Review Article

\title{
DIVERSITY AND DISTRIBUTION OF CRAB SPIDERS (THOMISIDAE: ARANEOMORPHAE: ARANEAE:ARACHNIDA) IN INDIA
}

\author{
Rajendra Singh ${ }^{1 *}$ and Garima Singh ${ }^{2}$ \\ ${ }^{1}$ Department of Zoology, Deendayal Upadhyay University of Gorakhpur, U.P., India \\ ${ }^{2}$ Department of Zoology, University of Rajasthan, Jaipur, Rajasthan, India
}

Article History: Received 19th May 2021; Accepted 20 $0^{\text {th }}$ June 2021; Published 30 2021

\begin{abstract}
The article deals with the faunal diversity of the crab spiders (Thomisidae: Araneomorphae: Araneae: Arachnida) in different states of India and the union territories. A total of 210 species belonging to 44 genera of Thomisidae was recorded in all states and the union territories of India except for Nagaland and Dadra \& Nagar Haveli and Daman \& Diu, out of which 143 species (68.4\%) were strictly endemic. However, among them, 6 species seem to be the case of erroneous report or misidentification. Two genera of crab spiders are highly speciose, e.g. Thomisus Walckenaer, 1805 (53 species) and Xysticus C.L. Koch, 1835 (25 species). A maximum of 74 species of these crab spiders was recorded from Maharashtra followed by 55 species from Madhya Pradesh, 52 species from West Bengal, 40 species from Kerala, 34 species each from Gujarat and Tamil Nadu, 33 species from Karnataka, 32 species from Chhattisgarh and Uttarakhand, 23 species from Meghalaya, 21 species each from Jammu \& Kashmir and Uttar Pradesh, 17 species from Assam, 14 species from Rajasthan, 12 species each from Andaman \& Nicobar Islands, Goa and Manipur, 11 species from Odisha and less than 10 species were recorded from other states. Seven species are considerably widely distributed, viz. Camaricus formosus Thorell, 1887 (17 states, 2 union territories), Indoxysticus minutus (Tikader, 1960) (16 states), Runcinia insecta (L. Koch, 1875) (15 states, 2 union territories), Thomisus projectus Tikader, 1960 (14 states, 1 union territories), Thomisus pugilis Stoliczka, 1869 (13 states, 2 union territories), Amyciaea forticeps (O. Pickard-Cambridge, 1873) (12 states, 1 union territory) and Thomisus lobosus Tikader, 1965 (12 states, 1 union territories). Several species of Thomisidae reported from India are recorded only from one state or from the type locality. Hence, intensive and extensive faunistic surveys for these spiders are required throughout the country.
\end{abstract}

Keywords: Thomisidae, Checklist, Faunal distribution, Crab spiders, India.

\section{INTRODUCTION}

The spiders (Araneae: Arthropoda: Chelicerata: Arachnida) are among the most frequent and abundant predators in the terrestrial ecosystem throughout the world. They not only devour lots of prey, mostly insects, but also serve as a food source for many carnivore animals (Nyffeler \& Birkhofer, 2017) and are very important component of the ecosystem. Their biodiversity ranks seventh in global diversity (49,483 species, 4,217 genera, 129 families, WSC, 2021) after Coleoptera, Lepidoptera, Hymenoptera, Diptera, Hemiptera and Acari (Singh et al., 2021). Except for few species, most of the spiders are not harmful to human beings; still, public opinion is not to their favour. Even the diversity studies are limited only in few localities. Despite recent researches on the faunistic biodiversity surveys of Indian spiders, their number is meagre and only 1867 species belonging to 475 genera are listed by Caleb \& Sankaran (2021) despite having very rich biodiversity and a tropical climate with biodiversity hotspots. Recently, the species distribution and checklist of 65 families of spiders have been updated in India (Akhilesh Sharma et al., 2020; Sharma et al., 2020; Singh et al., 2021; Singh, 2021; Singh, \& Singh, 2021; Rajendra Singh et al., 2020; Singh et al., 2020a, 2020b; . Singh et al., 2021; Tiwari et al., 2021; Tiwari \& Singh, 2021; Tiwari et al., 2021).

Thomisidae Sundevall, 1833 is an araneomorph, entelegyne (females have a genital plate), and stout ecribellate (spin sticky seizure silk) spider family which is 
distributed throughout the world. These spiders are commonly known as the crab spiders, flower spiders or flower crab spiders because of crab-like appearance. Presently, the family is the seventh largest family with 2155 known species in 170 genera after Salticidae (6346 species, 658 genera), Linyphiidae (4706 species, 620 genera) and Araneidae (3066 species, 177 genera), Gnaphosidae (2580 species, 163 genera), Theridiidae (2531 species, 124 genera) and Lycosidae (2431 species, 125 genera) (WSC, 2021). However, this diversity is only a fraction of its true diversity (Benjamin, 2011). The crab spiders have larger and stronger front legs than the other six and are held sideways and hold on to prey while paralysing it with a venomous bite. Like crabs, it can move sideways. They are cryptically coloured and usually 4-10 mm in length. The cephalothorax and abdomen of crab spiders are usually short and broad. Eight homogeneously coloured eyes are arranged in two transverse rows of four each, however, in life, the anterior median eyes appear lighter than the others. Other distinguishing characters are free chelicerae, bear two dentate tarsal claws on each laterigrade leg, one pair of book lungs and absence of cribellum and calamistrum (Tikader, 1971). The crab spiders also mimic ants of taxonomically diverse groups. Few species of crab spiders are social and mothers take care of spiderlings (Benjamin, Dimitrov, Gillespie, \& Hormiga, 2008). Most of the species are found on vegetation but some occur on dead bark, on the ground, or in leaf detritus. These spiders do not build web, rather capture the prey by ambushing and sometimes by active persuit. The crab spiders are not active hunters but are sit-and-wait predators that hunt in flowers, foliage, or leaf litter. They remain impassive until the prey arrives and catches it. Few crab spiders with flattened bodies either hunt in the crevices of tree trunks or under loose bark, or shelter under such crevices by day and come out at night to hunt (Tikader, 1971). Most of the species of Thomisidae are sexually dimorphic, males are sometimes much smaller than females. The females usually look after their egg sacs which are fastened to the vegetation until the spiderlings hatch. Benjamin (2011) studied the phylogeny of Thomisidae genera using morphological characters, the monophyly of internal groupings and subfamilies and explored the evolution of colour change behaviour and the eye arrangement patterns of the median ocular quadrangle in light of the preferred phylogenetic hypothesis.

The spiders play an important role in most terrestrial foodwebs and may be very abundant in several habitats. Moreover, despite the ecological significance of spiders in terrestrial ecosystems, they have received very little attention as far as their conservation is concerned, particularly for thomisid spiders. Among the thomisid spiders, only 2 species are listed as critically endangered in IUCN Red List of Threatened Animals: Bonapruncinia sanctaehelenae Benoit, 1977 (White et al., 2019) and Bassaniodes grohi (Wunderlich, 1992) (Cardoso et al., 2018) found only in St. Helena (territory of UK) and Madeira (an autonomous region of Portugal), respectively; and one species Firmicus insularis (Blackwall, 1877) found only in Seychelles is listed as endangered (Gerlach, 2014).
Information regarding the Indian Thomisidae is insufficient and highly incoherent primarily due to the unexplored diversity of these spiders in several parts of the country like other families of spiders (Singh, 2021a-e). There are several species of these spiders yet to be described and several species recorded from India have also been misidentified as they are said to be identified by using existing old literature without a re-examination of the corresponding type materials and without consulting any spider taxonomist. Hence, these reports need reexamination. At present the faunal survey results of crab spiders are all scattered in the literature and so far no updated consolidated account is available regarding their distribution pattern across the country. Therefore, this present work was carried out to make available an up-todate checklist of Indian Thomisidae like other families (Singh, 2021a-d; Singh \& Singh, 2021a, b; Tiwari et al., 2021a, b) in different states and the union territories of India.

\section{MATERIALS AND METHODS}

The present checklist is based on the published literature on spiders from India in recent past books, book chapters, journals, proceedings, records of Zoological Survey of India, Kolkata, few authentic theses, websites, and World Species Catalog (WSC, 2021) up to May 20, 2021. Some references to the faunistic surveys by Indian authors were omitted because of the repetition of the location. In most of the literature published earlier, several errors crept in the scientific names of the spiders even in the recent ones. This occurred because such information as soon becomes obsolete and, due to their perceived understanding, readers occasionally do not take into account new sources of data.

The researches on spider taxonomy like other taxa are continued with the description of new taxa, their modified status, and the publication of other nomenclatural decisions and clarifications (WSC, 2021; Singh, 2021a-e). If a spider species is identified only up to a generic level, it was considered as species if no other species of that genus is reported within that state. In the present checklist, attempts have been made to correct the errors in the scientific names of the spiders following WSC (2021). Only those synonymies were given that were reported in India, for other synonymies, WSC (2021) should be consulted. Only those species were considered endemic that were exclusively found only in India and such species are marked with $\left(^{*}\right)$. If the spider species is not endemic, its elsewhere distribution is also given following the WSC (2021) and other sources of literature. Seemingly, misidentified and erroneous records are marked with $(\dagger)$. The checklist includes species in alphabetical order. For each species, authority, year and distribution details of species in different states and union territories along with relevant references are given.

\section{RESULTS AND DISCUSSION}

In India, most probably Stoliczka (1869) was first to describe three species of crab spiders, Thomisus elongatus 
and Thomisus pugilis from West Bengal, and Phrynarachne peeliana from Assam. Thereafter, O. Pickard-Cambridge (1885) described three species, Tmarus dejectus and Xysticus mundulus from Jammu \& Kashmir; and Xysticus breviceps from Ladakh. In the same year, Simon (1885a, b) described Diaea pougneti (Simon, 1885b) and Ozyptila theobaldi (Simon, 1885b) from Tamil Nadu, and Stiphropus duriusculus (Simon, 1885a) from Karnataka. Later on, Thorell (1891) described Runcinia kinbergi and Thomisus armillatus from Andaman \& Nicobar Islands; Simon (1895a, b) described four species from Tamil Nadu (Dietopsa castaneifrons, Dietopsa parnassia, Holopelus malati, Lycopus trabeatus and Lysiteles catulus) and one species from Sikkim (Runcinia bifrons).

At the beginning of the $20^{\text {th }}$ century, Pocock (1901) described one species of crab spider, Angaeus pentagonalis from Tamil Nadu; and Leardi in Airaghi (1901a, b) recorded three species of crab spiders, one species from Uttarakhand, Thomisus dentiger (Thorell, 1887) and two species from Puducherry, Thomisus spectabilis Doleschall, 1859 and Xysticus cristatus (Clerck, 1757). Within five years, Simon (1906) described six species, three from Tamil Nadu (Oxytate chlorion, Thomisus leucaspis, Thomisus rigoratus); and one each from Puducherry (Tmarus fasciolatus), Kerala (Tmarus soricinus) and Himalayan plateaus (Loxobates castetsi). After a decade, Sherriffs (1929) recorded nine species of crab spiders from Andaman \& Nicobar Islands (1 species), Karnataka (1 species), Tamil Nadu (6 species) and West Bengal (1 species). After about three decades, Reimoser (1934) described Runcinia escheri from Tamil Nadu and Caporiacco (1935) described and recorded few species of crab spiders from Jammu \& Kashmir. Among the Indian authors, Tikader (1960) was the first to describe six species of crab spiders, two species each from Assam (Indoxysticus minutes, Thomisus projectus) and Gujarat (Thomisus bulani, Thomisus dhakuriensis) and one species each from Chhattisgarh (Synema decoratum) and Meghalaya (Ozyptila khasi). Later, several authors (Tikader, 1961, 1962, 1963, 1964, 1965, 1966a, b, 1968, 1970, 1971, 1980; Basu, 1963, 1964a, b, 1965a, b, 1979; Sen, 1963, 1964; Sen \& Basu, 1963; Tikader \& Biswas, 1974, 1979; Biswas, 1977; Biswas \& Mazumder, 1981; Gajbe \& Rane, 1992; Reddy \& Patel, 1992; Kumari \& Mittal, 1994, 1997, 1999; Gajbe \& Gajbe, 1999a, b, 2000a, b) have described and recorded about one hundred species of crab spiders from several parts of India. Long back, Tikader $(1971,1980)$ had nicely illustrated and documented the Thomisidae of India. In the present century, several workers have described 41 species of crab spiders from India, 25 species from Madhya Pradesh (Bhandari \& Gajbe, 2001; Gajbe, 2004a, 2004d, 2005, 2008a, b); 8 species from West Bengal (Saha \& Raychaudhuri, 2004, 2007a, b; Sen et al., 2010b, 2012); 3 species from Kerala (Patel, 2003a; Biswas \& Roy, 2005a, b); 2 species from Tamil Nadu (Benjamin, 2017); and one species each from Andhra Pradesh (Rao et al., 2006), Maharashtra (Bastawade, 2002) and Telangana (Pravalikha \& Srinivasulu, 2015). In addition, in the present century, several workers have recorded many species of Thomisidae across India in survey programmes.
At present, 210 species belonging to 44 genera were described or recorded from India during the last 153 years (1869-2021), out of which, 143 species (68.1\%) were strictly endemic, i.e. described and recorded only in India while few more species were described from India but were also later recorded in neighbouring countries. However, the Indian record is only $9.7 \%$ of the world Thomisidae fauna (2154 species placed in 169 genera (WSC, 2021). All these spiders were distributed in all the Indian states except Nagaland and Dadra and Nagar Haveli and Daman and Diu (Figure 1). Six species marked with $(\dagger)$ seem to be erroneous report or the case of misidentification. One species, Misumena oblonga O. Pickard-Cambridge, 1885 was collected by its author from Murree (Pakistan) to Sind Valley (Jammu \& Kashmir), however, its distribution is mentioned in Yarkand (China) by WSC (2021). Similarly, Runcinia spinulosa O. Pickard-Cambridge (1885) was recorded only from Murree (Pakistan) but it is included in the checklists of Indian spiders (Siliwal et al., 2005; Keswani et al., 2012; Caleb \& Sankaran, 2021; WSC, 2021). This species is excluded from this checklist. Surprisingly, one species of crab spider, Misumena chrysanthemi Sebastian \& Peter, 2009 which is an invalid species (Zschokke \& Logunov, 2010; WSC, 2021), still it was identified and recorded by several workers in different states (Assam-Singh et al., 2012; Pandit, 2019; BiharPriyadarshini et al., 2015; Kerala-Asima et al., 2020; Maharashtra-Nerlekar et al., 2016). Caleb \& Sankaran (2021) recently enlisted only 181 species of Thomisidae described under 40 genera and the rest of the species recorded in India are either overlooked by them or are cases of misidentification and erroneous reports.

Two genera of crab spiders are highly speciose, e.g. Thomisus Walckenaer, 1805 (53 species) and Xysticus C.L. Koch, 1835 (25 species). A maximum of 74 species of these crab spiders was recorded from Maharashtra followed by 55 species from Madhya Pradesh, 52 species from West Bengal, 40 species from Kerala, 34 species each from Gujarat and Tamil Nadu, 33 species from Karnataka, 32 species from Chhattisgarh and Uttarakhand, 23 species from Meghalaya, 21 species each from Jammu \& Kashmir and Uttar Pradesh, 17 species from Assam, 14 species from Rajasthan, 12 species each from Andaman \& Nicobar Islands, Goa and Manipur, 11 species from Odisha and less than 10 species were recorded from other states (Figure 1). No species of crab spider is recorded from Nagaland and Dadra and Nagar Haveli and Daman and Diu. Surprisingly, a very poor record of these spiders was in Andhra Pradesh, Bihar, Himachal Pradesh, Jharkhand, and Telangana. Indeed, no survey was conducted in these regions and hence, it requires an intensive and extensive faunistic survey in these areas. Most of the surveys were conducted in Madhya Pradesh, Gujarat, Maharashtra, Kerala, Tamil Nadu and West Bengal. Out of 210 species of Thomisidae recorded from India, 7 species are considerably widely distributed, viz. Camaricus formosus Thorell, 1887 (17 states, 2 union territories), Indoxysticus minutus (Tikader, 1960) (16 states), Runcinia insecta (L. Koch, 1875) (15 states, 2 union territories), Thomisus projectus Tikader, 1960 (14 states, 1 union territories), Thomisus pugilis 
Stoliczka, 1869 (13 states, 2 union territories), Amyciaea forticeps (Opickard-Cambridge, 1873) (12 states, 1 union territory) and Thomisus lobosus Tikader, 1965 (12 states, 1 union territories). Several species of Thomisidae reported from India are recorded only from one state or from the type locality. Endemism in thomisid spiders in India is high at the species level (143 species out of 210 species recorded), and these species are threatened with the loss and fragmentation of habitats. Though none of the species of thomisid spider is listed in IUCN Red List of Threatened Animals as endangered or vulnerable in India, still like others, they are also threatened due to habitat loss and fragmentation and other anthropogenic activities hampering ecosystem. Therefore, conservation efforts are immediately needed for their conservation practices. Results demonstrated that most of the surveys conducted in India have remained restricted to a few states and union territories particularly, in the Western Ghats, Eastern Ghats and north and northeast India, and most areas in the country still remain virgin. Hence, a systematic faunistic surveys for these crab spiders are essential for the entire country to find an overall representation of thomisid spiders in the country. Following is the detailed list of these spiders arranged alphabetically which are distributed in Indian states and union territories.

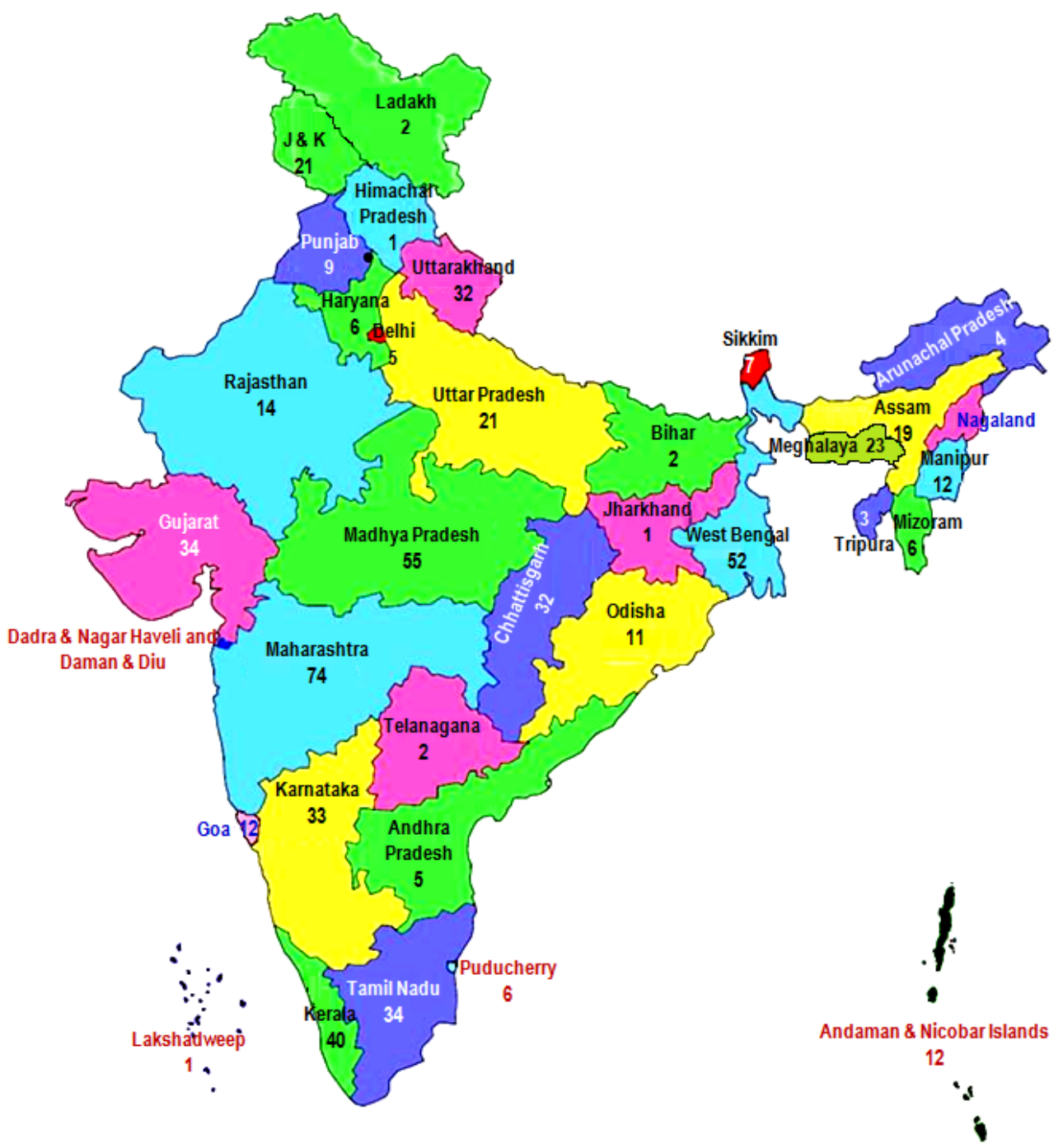

Figure 1. Number of species of the spider family Thomisidae in Indian states and union territories.

\section{Checklist of Crab Spiders (Thomisidae) in Indian States and Union Territories}

\section{Amyciaea albomaculata (O. Pickard-Cambridge, 1874)†}

- Kerala (Sumesh \& Sudhikumar, 2020)
Elsewhere: Australia, New Guinea

2. Amyciaea forticeps (O. Pickard- Cambridge, 1873)

- Assam (Chetia \& Kalita, 2012; Gupta et al., 2015)

- Chhattisgarh (Gajbe \& Sharma, 1994)

- Goa (Pandit \& Dharwadkar, 2020) 
- Gujarat (Patel, 2003b; Siliwal et al., 2003a; Solanki et al., 2020)

- Karnataka (Nalini Bai \& Ravindranatha, 2012; Mubeen \& Basavarajappa, 2018)

- Kerala (Sebastian et al., 2005a; Sunil Jose et al., 2003; Rajeevan et al., 2019; Sumesh \& Sudhikumar, 2020)

- Maharashtra (Tikader, 1971, 1980; Gajbe, 1995b; Rithe, 2012; More \& Sawant, 2013)

- Manipur (Kananbala et al., 2018)

- Meghalaya (Bhattacharya et al., 2017)

- Odisha (Choudhury et al., 2019)

- Puducherry (Simon, 1906)

- Tamil Nadu (Kadam \& Rajkumar, 2020)

- Uttarakhand (Gupta \& Siliwal, 2012)

- West Bengal (Bhattacharya, 1934; Biswas \& Biswas, 1992; Sen et al., 2015)

Elsewhere: China, Malaysia

3. Amyciaea lineatipes O. Pickard-Cambridge, 1901

- Tripura (Dey et al., 2013)

Elsewhere: Indonesia, Singapore

4. Amyciaea sp.

- Karnataka (Prashanthakumara \& Venkateshwarlu, 2017)

- Odisha (Choudhury et al., 2019)

5. Angaeus pentagonalis Pocock, 1901*

Syn. Misumenoides kripalani Tikader, 1963

- Andaman \& Nicobar Islands (Tikader, 1977, 1980)

- Karnataka (Tikader, 1963, 1971, 1977a, 1980)

- Maharashtra (Tikader, 1977)

- Tamil Nadu (Pocock, 1901; Karthikeyani et al., 2017)

6. Angaeus sp.

- Goa (Pandit \& Dharwadkar, 2020)

- Karnataka (Abhijith, 2019)

7. Bomis bengalensis Tikader, 1962*

- West Bengal (Tikader, 1962, 1971, 1980; Tikader \& Biswas, 1981; Biswas \& Biswas, 1992)

8. Bomis calcuttaensis Biswas \& Mazumder, 1981*

- West Bengal (Biswas \& Mazumder, 1981; Biswas \& Biswas, 1992; Majumder, 2005)

9. Bomis khajuriai Tikader, 1980*

- Madhya Pradesh (Tikader, 1980; Sen et al., 2010b)

- Tamil Nadu (Caleb, 2020a)

10. Bomis sp.

- Karnataka (Abhijith, 2019)

- Odisha (Panda et al., 2011; Mohapatra et al., 2014)

- Rajasthan (Kashmeera et al., 2020)

- Uttarakhand (Gupta \& Siliwal, 2012)

\section{Borboropactus bituberculatus Simon, 1884}

- Andaman \& Nicobar Islands (Dash \& Sivaperuman, 2021a)
Elsewhere: China, Indonesia, Papua New Guinea

12. Borboropactus elephantus (Tikader, 1966)*

Syn. Regillus elephantus Tikader, 1966

- Assam (Tikader, 1966a)

- Maharashtra (Tikader, 1980)

- Meghalaya (Tikader, 1971, 1980; Biswas \& Majumder, 1995)

13. Camaricus bipunctatus Bastawade, 2002*

- Andhra Pradesh (Bastawade \& Khandal, 2006)

- Arunachal Pradesh (Bastawade, 2002; Bastawade \& Khandal, 2006)

- Maharashtra (Bastawade, 2002; Bastawade \& Khandal, 2006; More \& Sawant, 2013; More, 2015a)

14. Camaricus formosus Thorell, 1887

Syn. Camaricus fornicatus Thorell, 1890

- Andaman \& Nicobar Islands (Tikader, 1977, 1980; Tikader \& Biswas, 1981; Majumder, 2004, 2005)

- Arunachal Pradesh (Biswas \& Biswas, 2006)

- Assam (Chetia \& Kalita, 2012; Singh et al., 2012; Gupta et al., 2015; Ahmed, 2018)

- Delhi (Malik et al., 2015)

- Goa (Bastawade \& Borkar, 2008; Pandit \& Dharwadkar, 2020)

- Gujarat (Chandra et al., 2021)

- Karnataka (Tikader \& Biswas, 1981; Majumder, 2004, 2005)

- Kerala (Ambily \& Antony, 2016; Jose et al., 2018; Rajeevan et al., 2019)

- Madhya Pradesh (Sen et al., 2010b)

- Maharashtra (Tikader \& Biswas, 1981; Majumder, 2004; More \& Sawant, 2013)

- Manipur (Biswas \& Biswas, 2004; Kananbala et al., 2018)

- Meghalaya (Bhattacharya et al., 2017)

- Mizoram (Biswas \& Biswas, 2007)

- Odisha (Majumder, 2005; Panda et al., 2011; Mohapatra et al., 2014; De \& Palita, 2018)

- Tamil Nadu (Bastawade \& Borkar, 2008; Karthikeyani, 2013)

- Tripura (Dey et al., 2013)

- Uttar Pradesh (Singh \& Singh, 2014; Sharma \& Singh, 2018a, b)

- Uttarakhand (Gupta \& Siliwal, 2012)

- West Bengal (Tikader, 1980; Tikader \& Biswas, 1981; Biswas \& Biswas, 1992; Majumder, 2004, 2005; Saha \& Raychaudhuri, 2007a; Sen et al., 2015; Saha et al., 2016)

Elsewhere: Bangladesh, China, Indonesia, Malaysia, Myanmar, Philippines

15. Camaricus khandalaensis Tikader, 1980*

- Gujarat (Siliwal et al., 2003a; Yadav et al., 2017a) 
- Kerala (Sudhikumar et al., 2005; Sunil Jose et al., 2008; Joseph et al., 2017)

- Maharashtra (Tikader, 1980; Pande et al., 2013)

- Odisha (Choudhury et al., 2019)

- West Bengal (Saha \& Raychaudhuri, 2007a)

16. Camaricus maugi (Walckenaer, 1837)

- Tripura (Dey et al., 2013)

Elsewhere: Indonesia, Myanmar, Vietnam

17. Camaricus rinkae Biswas \& Roy, 2005*

- Kerala (Biswas \& Roy, 2005b)

18. Camaricus siltorsus Saha \& Raychaudhuri, 2007*

- West Bengal (Saha \& Raychaudhuri, 2007a; Sen et al., 2015)

19. Camaricus sp.

- Andhra Pradesh (Palem et al., 2016)

- Karnataka (Bhat et al., 2013)

- Odisha (Mohapatra et al., 2014)

- Tamil Nadu (Kadam \& Rajkumar, 2020)

- Uttarakhand (Uniyal et al., 2011)

20. Demogenes andamanensis (Tikader, 1980)*

Syn. Misumenops andamanensis Tikader, 1980)

- Andaman \& Nicobar Islands (Tikader, 1980)

- Kerala (Sudhikumar et al., 2005; Sunil Jose et al., 2008; Joseph et al., 2017)

21. Diaea bengalensis Biswas \& Mazumder, 1981*

- Maharashtra (Rithe, 2012; More \& Sawant, 2013)

- Manipur (Biswas \& Biswas, 2004; Kananbala et al., 2018)

- West Bengal (Biswas \& Mazumder, 1981; Biswas \& Biswas, 1992; Majumder, 2005).

22. Diaea dorsata (Fabricius, 1777)

- Manipur (Kananbala et al., 2018)

Elsewhere: Caucasus, Europe, Iran, Japan, Russia, Turkey

23. Diaea pougneti Simon, 1885*

- Tamil Nadu (Simon, 1885b; Karthikeyani et al., 2017; Caleb \& Karthikeyani, 2020)

\section{Diaea sp.}

- Gujarat (Parmar, 2018)

- Uttarakhand (Uniyal et al., 2011)

25. Diaea subdola Pickard-Cambridge, 1885

Syn. Misumena horai Tikader, 1968

- Jammu \& Kashmir (Caporiacco, 1935)

- Meghalaya (Tikader, 1968, 1971, 1980; Biswas \& Majumder, 1995)

- Uttar Pradesh (Hore \& Uniyal, 2008a, b; Uniyal \& Hore, 2009)

Elsewhere: China, Formosa, Japan, Korea, Russia

26. Dietopsa castaneifrons (Simon, 1895)*

Syn. Dietopsis castaneifrons Simon, 1895
- Tamil Nadu (Simon, 1895b)

27. Dietopsa parnassia (Simon, 1895)*

Syn. Dietopsis parnassia Simon, 1895)

- Tamil Nadu (Simon, 1895a, 1906; Karthikeyani et al., 2017; Caleb \& Karthikeyani, 2020)

28. Ebrechtella concinna (Thorell, 1877)

Syn. Diaea subargentata O. Pickard-Cambridge, 1885; Misumena silveryi Tikader, 1965; Misumenops maygitgitus Barrion \& Litsinger, 1995)

- Gujarat (Parmar, 2013; Parmar \& Acharya, 2015)

- Jammu \& Kashmir (Caporiacco, 1935)

- Kerala (Sunil Jose et al., 2008; Mathew et al., 2014)

- Maharashtra (Tikader, 1965, 1971, 1980)

- Mizoram (Chowdhury et al., 2017)

Elsewhere: Indonesia, New Guinea, Pakistan, Philippines

29. Ebrechtella sp.

- Kerala (Joseph et al., 2017; Sumesh \& Sudhikumar, 2020)

30. Ebrechtella sufflava (O. Pickard-Cambridge, 1885)

Syn. Diaea sufflava Pickard-Cambridge, 1885; Misumena expallidata Pickard-Cambridge, 1885

- Jammu \& Kashmir (Caporiacco, 1935)

Elsewhere: Pakistan

31. Ebrechtella tricuspidata (Fabricius, 1775)

Syn. Misumena tricuspidata (Fabricius, 1775)

- Jammu \& Kashmir (Caporiacco, 1935)

- Maharashtra (Chapke, 2012)

Elsewhere: Caucasus, Central Asia, China, Europe, Iran, Japan, Kazakhstan, Korea, Pakistan, Russia, Turkey

32. Epidius armatus (Thorell, 1895)

Syn. Platythomisus bazarus Tikader, 1970; Epidius bazarus (Tikader, 1970)

- Kerala (Joseph et al., 2017)

- Sikkim (Tikader, 1970, 1971, 1980)

- Tamil Nadu (Benjamin, 2017)

- West Bengal (Tang et al., 2009)

Elsewhere: China, Laos, Myanmar

33. Epidius longimanus Benjamin, 2017*

- Tamil Nadu (Benjamin, 2017)

34. Epidius longipalpis Thorell, 1877

- Kerala (Joseph et al., 2017)

Elsewhere: Indonesia, Sri Lanka

35. Epidius mahavira Benjamin, 2017*

- Tamil Nadu (Benjamin, 2017)

36. Epidius parvati Benjamin, 2000

- Kerala (Joseph et al., 2017; Malamel \& Sudhikumar, 2017, 2020)

37. Henriksenia hilaris (Thorell, 1877)

Syn. Misumenoides deccanes Tikader, 1965; Misumenoides shulli Tikader, 1965; Diaea jaintious Tikader, 1966; 
Misumena decorata Tikader, 1980; Misumena braminica Simon, 1906

- Assam (Tikader, 1966a)

- Kerala (Sudhikumar et al., 2005; Sunil Jose et al., 2008; Adarsh \& Nameer, 2016)

- Maharashtra (Tikader, 1965, 1980; Meshram, 2011; Pande et al., 2013)

- Meghalaya (Tikader, 1971, 1980; Biswas \& Majumder, 1995)

- Puducherry (Simon, 1906)

- Tamil Nadu (Simon, 1906; Karthikeyani et al., 2017; Caleb \& Karthikeyani, 2020; Caleb, 2020a)

- Uttarakhand (Tikader, 1965, 1971, 1980; Biswas \& Biswas, 2010; Uniyal et al., 2011)

- West Bengal (Ghosh et al., 2018)

Elsewhere: Bangladesh, China, Indonesia, Malaysia, New Guinea, Philippines.

\section{Heriaeus horridus Tyschchenko, 1965}

Syn. Misumenops kumaonensis Tikader, 1980

- Maharashtra (Bastawade \& Khandal, 2006; Warghat et al., 2011)

- Uttarakhand (Tikader, 1980)

Elsewhere: Central Asia, Russia, Ukraine

39. Holopelus malati Simon, 1895 *

- Tamil Nadu (Simon, 1895b)

40. Indosmodicinus bengalensis Sen et al., 2010

- West Bengal (Sen et al., 2010a, 2015)

Elsewhere: China

41. Indoxysticus lumbricus Tang $\mathrm{Li}, 2010 †$

- Gujarat (Yadav, 2019)

Elsewhere: China

42. Indoxysticus minutus (Tikader, 1960)

Syn. Xysticus minutus Tikader, 1960

- Assam (Chetia \& Kalita, 2012; Das et al., 2015)

- Bihar (Priyadarshini et al., 2015, 2018)

- Chhattisgarh (Gajbe \& Sharma, 1994)

- Gujarat (Tikader, 1971, 1980; Tikader \& Biswas, 1981; Siliwal et al., 2003a, b; Gajbe, 2004d; Majumder, 2005; Parmar, 2013; Parmar \& Acharya, 2015; Yadav et al., 2017a)

- Karnataka (Bhat et al., 2013; Vaibhav et al., 2017; Nijagal et al., 2020)

- Kerala (Jose et al., 2018; Asima et al., 2020; Sumesh \& Sudhikumar, 2020)

- Madhya Pradesh (Gajbe, 2003a, b, 2004d, 2007; Chetia \& Kalita, 2012)

- Maharashtra (Tikader, 1971; Tikader \& Biswas, 1981; Gajbe, 2004d, 2007; Majumder, 2005; Meshram, 2011; Vairale \& Wagh, 2021)

- Meghalaya (Tikader, 1968, 1971, 1980; Tikader \& Biswas, 1981; Gajbe, 2004d; Majumder, 2005)
- Odisha (Choudhury et al., 2019)

- Punjab (Kumari, 1983)

- Rajasthan (Chauhan et al., 2009; Lawania \& Trigunayat, 2015; Kumari et al., 2017)

- Tamil Nadu (Caleb, 2020a)

- Uttar Pradesh (Lawania \& Mathur, 2014a; Kumar et al., 2017a; Kadam \& Rajkumar, 2020)

- Uttarakhand (Uniyal et al., 2011; Gupta \& Siliwal, 2012)

- West Bengal (Tikader, 1960, 1971, 1980; Tikader \& Biswas, 1981; Biswas \& Biswas, 1992; Gajbe, 2004d; Majumder, 2005; Sen et al., 2015)

Elsewhere: Sri Lanka

43. Indoxysticus sp.

- Odisha (Choudhury et al., 2019)

44. Loxobates castetsi (Simon, 1906)*

Syn. Oxylate castetsi Simon, 1906

- Himalayan plateaus (Simon, 1906)

- Karnataka (Bhat et al., 2013)

45. Loxobates kapuri (Tikader, 1980)*

Syn. Dieta kapuri Tikader, 1980

- Maharashtra (Tikader, 1980)

46. Loxobates sp.

- Karnataka (Bhat et al., 2013)

47. Simon, 1895*

- Tamil Nadu (Simon, 1895b, 1906; Karthikeyani et al., 2017)

48. Lysiteles brunettii (Tikader, 1962)*

Syn. Synaema brunettii Tikader, 1962

- Madhya Pradesh (Gajbe, 2003b, 2007)

- Uttarakhand (Uniyal et al., 2011)

- West Bengal (Tikader, 1962, 1971, 1980; Biswas \& Biswas, 1992; Gajbe, 2007)

49. Lysiteles catulus Simon, 1895*

- Tamil Nadu (Simon, 1895b, 1906)

50. Lysiteles excultus (O. Pickard-Cambridge, 1885)

Syn. Synema exculta O. Pickard-Cambridge, 1885

- Jammu \& Kashmir (Caporiacco, 1935)

Elsewhere: Pakistan

51. Lysiteles mandali (Tikader, 1966)

Syn. Xysticus mandali Tikader, 1966

- Assam (Tikader, 1966a)

- Meghalaya (Tikader, 1968, 1971, 1980; Biswas \& Majumder, 1995)

Elsewhere: China

52. Lysiteles niger Ono, 1979

- Uttarakhand (Uniyal et al., 2011)

Elsewhere: Bhutan, China, Nepal

53. Lysiteles sp.

- Uttar Pradesh (Kumar et al., 2017a) 
- Uttarakhand (Quasin \& Uniyal, 2010; Uniyal et al., 2011)

\section{Massuria roonwali (Basu, 1964)*}

Syn. Pistius roonwali Basu, 1964.

- Uttar Pradesh (Sen et al., 2015)

- Uttarakhand (Basu, 1964a; Tikader, 1971, 1980; Gupta \& Siliwal, 2012)

- West Bengal (Saha \& Raychaudhuri, 2007a; Sen et al., 2015)

55. Massuria sp.

- Goa (Pandit \& Dharwadkar, 2020)

56. Massuria sreepanchamii (Tikader, 1962)*

Syn. Pistius sreepanchamii Tikader, 1962

- Manipur (Kananbala et al., 2018)

- Meghalaya (Tikader, 1962, 1971, 1980; Biswas \& Majumder, 1995)

- West Bengal (Saha \& Raychaudhuri, 2007a)

57. Massuria uthoracica Sen et al., 2012*

- West Bengal (Sen et al., 2012, 2015)

58. Mastira menoka (Tikader, 1963)*

Syn. Misumena menoka Tikader, 1963

- Gujarat (Yadav et al., 2017a)

- Karnataka (Tikader, 1963, 1980)

- Kerala (Lehtinen, 2004)

- Maharashtra (Meshram, 2011)

- Uttar Pradesh (Sharma \& Singh, 2018a, b)

- Uttarakhand (Uniyal et al., 2011; Pooja et al., 2019)

59. Mastira nicobarensis (Tikader, 1980)*

Syn. Mesumena nicobarensis Tikader, 1980

- Andaman \& Nicobar Islands (Tikader, 1980)

- West Bengal (Saha \& Raychaudhuri, 2007a; Sen et al., 2015)

60. Mecaphesa celer (Hentz, 1847)†

Syn. Misumenops celer (Hentz, 1847)

- Rajasthan (Lawania \& Mathur, 2017)

Elsewhere: Canada, Mexico, USA

61. Misumena annapurna Tikader, 1963*

- Karnataka (Tikader, 1963, 1971, 1980)

- Maharashtra (Tikader, 1971, 1980; Rithe, 2012)

62. Misumena ganpatii Kumari \& Mittal, 1994*

- Punjab (Kumari \& Mittal, 1994)

63. Misumena greenae Tikader, 1965*

- Maharashtra (Tikader, 1965, 1971, 1980; More \& Sawant, 2013)

64. Misumena indra Tikader, 1963*

- Karnataka (Tikader, 1963, 1971, 1980)

- Maharashtra (More \& Sawant, 2013; More, 2015a)

- Uttar Pradesh (Hore \& Uniyal, 2008a, b; Uniyal \& Hore, 2009)
65. Misumena mickeyi Sen, Saha \& Raychaudhuri, 2012*

- West Bengal (Sen et al., 2012, 2015)

66. Misumena mridulai Tikader, 1962*

- Kerala (Sunil Jose \& Sebastian, 2001)

- Maharashtra (Meshram, 2011; Warghat et al., 2011)

- Meghalaya (Tikader, 1962, 1971, 1980; Biswas \& Majumder, 1995)

- Uttar Pradesh (Hore \& Uniyal, 2008a, b; Uniyal \& Hore, 2009)

- Uttarakhand (Uniyal et al., 2011)

67. Misumena oblonga O. Pickard-Cambridge, 1885

- Jammu \& Kashmir (Pickard-Cambridge, 1885; Caporiacco, 1935)

68. Misumena ritujae Gajbe, 2008*

- Madhya Pradesh (Gajbe, 2008a)

- Maharashtra (Rithe, 2012)

69. Misumena sp.

- Assam (Das et al., 2015; Saha et al., 2015a)

- Gujarat (Parasharya \& Pathan, 2013; Parmar et al., 2015)

- Kerala (Patel, 2003a; Sunil Jose et al., 2008; Sebastian et al., 2011)

- Ladakh (Uniyal, 2006)

- Maharashtra (Lanka et al., 2017)

- Manipur (Kananbala et al., 2018)

- Punjab (Kumari, 1983)

- Uttarakhand (Uniyal et al., 2011; Gupta \& Siliwal, 2012)

70. Misumena vatia (Clerck, 1757)

- Assam (Chetia \& Kalita, 2012)

- Goa (Halarnkar \& Pai, 2018)

- Karnataka (Mubeen \& Basavarajappa, 2018; Nijagal et al., 2020)

- Meghalaya (Bhattacharya et al., 2017)

- Uttar Pradesh (Kumar et al., 2017a, b)

Elsewhere: Caucasus, Central Asia, China, Europe, Iran, Japan, Kazakhstan, Korea, North America, Russia, Turkey

71. Misumenoides gwarighatensis Gajbe, 2004*

- Chhattisgarh (Kujur \& Ekka, 2012; Ekka \& Kujur, 2015).

- Madhya Pradesh (Gajbe, 2004d; Patil, 2012)

- Maharashtra (Rithe, 2012)

72. Misumenoides naginae Biswas \& Roy, 2008*

- Uttarakhand (Biswas \& Roy, 2008)

73. Misumenoides sp.

- Chhattisgarh (Kujur \& Ekka, 2016)

- Gujarat (Parasharya \& Pathan, 2013)

- Karnataka (Prashanthakumara \& Venkateshwarlu, 2017)

- Madhya Pradesh (Gajbe, 2003a, b)

74. Misumenops khandalaensis Tikader, 1965* 
- Maharashtra (Tikader, 1965, 1971, 1980; Bastawade \& Khandal, 2006; Meshram, 2011; More \& Sawant, 2013)

75. Misumenops rubrodecoratus Millot, 1942†

- Karnataka (Shraddha \& Chaturved, 2019)

Elsewhere: Africa

76. Misumenops sp.

- Karnataka (Abhijith, 2019)

- Kerala (Sunil Jose et al., 2008)

- Uttarakhand (Uniyal et al., 2011)

77. Misumessus oblongus (Keyserling, 1880)†

- Karnataka (Tabasum et al., 2018)

Elsewhere: Canada, USA

78. Monaeses jabalpurensis Gajbe \& Rane, 1992*

- Chhattisgarh (Kujur \& Ekka, 2012; Ekka \& Kujur, 2015)

- Madhya Pradesh (Gajbe \& Rane, 1992; Gajbe, 2003a, b, 2004d, 2007; Patil, 2012)

\section{Monaeses mukundi Tikader, 1980*}

- Gujarat (Patel, 2003b; Yadav et al., 2017a)

- Maharashtra (Tikader, 1980; Pande et al., 2013)

80. Monaeses pachpediensis (Tikader, 1980)*

Syn. Tmarus pachpediensis Tikader, 1980

- Madhya Pradesh (Tikader, 1980; Gajbe, 2003b)

- Maharashtra (Warghat et al., 2014; Vairale \& Wagh, 2021)

81. Monaeses parvati Tikader, 1963*

- Delhi (Malik et al., 2015)

- Gujarat (Siliwal et al., 2003b; Yadav et al., 2017a)

- Maharashtra (Tikader, 1963, 1971, 1980)

- Punjab (Tikader, 1971)

\section{Monaeses sp.}

- Chhattisgarh (Kujur \& Ekka, 2016).

- Gujarat (Parasharya \& Pathan, 2013)

- Uttarakhand (Gupta \& Siliwal, 2012)

83. Oxytate chlorion (Simon, 1906)*

Syn. Dieta chlorion Simon, 1906

- Tamil Nadu (Simon, 1906; Leardi in Airaghi, 1901b; Karthikeyani et al., 2017)

84. Oxytate elongata (Tikader, 1980)*

Syn. Dieta elongata Tikader, 1980

- Assam (Das et al., 2015)

- Chhattisgarh (Kujur \& Ekka, 2012; Ekka \& Kujur, 2015)

- Delhi (Malik et al., 2015)

- Gujarat (Patel, 2003b; Siliwal et al., 2003a; Yadav et al., 2017a)

- Madhya Pradesh (Gajbe, 2003b, 2004d, 2005; Patil, 2012)

- Maharashtra (Tikader, 1980; Gajbe, 2004d, Gajbe, 2005; Bastawade \& Khandal, 2006; Rithe, 2012; More \& Sawant, 2013; More, 2015a)
- Manipur (Kananbala et al., 2018)

- Uttarakhand (Gupta \& Siliwal, 2012)

85. Oxytate greenae (Tikader, 1980)*

Syn. Dieta greenae Tikader, 1980

- Andaman \& Nicobar Islands (Tikader, 1980)

- Kerala (Dhali et al., 2019; Sumesh \& Sudhikumar, 2020)

- West Bengal (Sen et al., 2015)

86. Oxytate kanishkai (Gajbe, 2008)*

Syn. Dieta kanishkai Gajbe, 2008

- Madhya Pradesh (Gajbe, 2008b)

Recently, this species was inadvertently placed in Philodromidae (its earlier placement) by Singh \& Singh (2021).

87. Oxytate virens (Thorell, 1891)

- Assam (Chetia \& Kalita, 2012; Das et al., 2015; Ahmed, 2018)

- Gujarat (Parmar, 2013; Parmar et al., 2015)

- Karnataka (Bhat et al., 2013)

- Kerala (Mathew et al., 2014; Ambily \& Antony, 2016; Jose et al., 2018; Rajeevan et al., 2019)

- Maharashtra (Nerlekar et al., 2016; Lanka et al., 2017)

- Manipur (Kananbala et al., 2018)

- Mizoram (Chowdhury et al., 2017)

- Tamil Nadu (Karthikeyani et al., 2017; Kadam \& Rajkumar, 2020)

Elsewhere: Singapore, Vietnam.

88. Oxytate sp.

- Assam (Chetia \& Kalita, 2012; Pandit, 2019)

- Goa (Pandit \& Dharwadkar, 2020)

- Gujarat (Parmar \& Patel, 2017)

- Odisha (Choudhury et al., 2019)

89. Ozyptila amkhasensis Tikader, 1980*

Syn. Oxyptila amkhasensis Tikader, 1980

- Chhattisgarh (Kujur \& Ekka, 2016)

- Madhya Pradesh (Tikader, 1980; Gajbe, 2003b, 2004d; Patil, 2012)

90. Ozyptila brevipes (Hahn, 1826)

- Rajasthan (Kashmeera et al., 2020)

Elsewhere: Europe, Russia

91. Ozyptila chandosiensis Tikader, 1980*

Syn. Oxyptila chandosiensis Tikader, 1980

- Maharashtra (More \& Sawant, 2013; More, 2015a)

- Rajasthan (Sivaperuman \& Rathore, 2004)

- Uttar Pradesh (Tikader, 1980)

92. Ozyptila jabalpurensis Bhandari \& Gajbe, 2001* Syn. Oxyptila jabalpurensis Bhandari \& Gajbe, 2001

- Chhattisgarh (Kujur \& Ekka, 2012; Ekka \& Kujur, 2015)

- Madhya Pradesh (Bhandari \& Gajbe, 2001; Gajbe, 2003b, 2004a; Patil, 2012) 
93. Ozyptila khasi Tikader, 1960*

Syn. Oxyptila khasi Tikader, 1960

- Meghalaya (Tikader, 1961, 1971, 1980; Biswas \& Majumder, 1995; )

- West Bengal (Sen et al., 2015; Raychaudhuri et al., 2016; Saha et al., 2016)

94. Ozyptila manii Tikader, 1961*

Syn. Oxyptila manii Tikader, 1961

- Assam [Saha et al., 2015a]

- Uttar Pradesh (Hore \& Uniyal, 2008a, b; Uniyal \& Hore, 2009)

- West Bengal (Tikader, 1961, 1971, 1980; Biswas \& Biswas, 1992)

95. Ozyptila maratha Tikader, 1971*

Syn. Oxyptila maratha Tikader, 1971; Oxyptila gertschi Tikader, 1964)

- Gujarat (Patel \& Pillai, 1988; Yadav et al., 2017a)

- Madhya Pradesh (Tikader, 1980)

- Maharashtra (Tikader, 1964, 1971, 1980; Meshram, 2011; Rithe, 2012; More, 2015a).

96. Ozyptila reenae Basu, 1964*

Syn. Oxyptila reenae Basu, 1964

- Rajasthan (Kashmeera et al., 2020)

- West Bengal (Basu, 1964b; Tikader, 1971, 1980; Biswas \& Biswas, 1992)

97. Ozyptila theobaldi Simon, 1885*

Syn. Oxyptila theobaldi Simon, 1885

- Tamil Nadu (Simon, 1885b, 1906; Karthikeyani et al., 2017)

98. Ozyptila trux (Blackwall, 1846)†

- Jammu \& Kashmir (Punjoo \& Bhat, 2015)

Elsewhere: Canada, Caucasus, Europe, Japan, Russia

99. Ozyptila sp.

- Gujarat (Parasharya \& Pathan, 2013)

- Kerala (Sudhikumar et al., 2005; Sunil Jose et al., 2008)

- Maharashtra (Lanka et al., 2017)

- Mizoram (Chowdhury et al., 2017)

- Uttar Pradesh (Hore \& Uniyal, 2008a, b; Uniyal \& Hore, 2009)

- Uttarakhand (Uniyal et al., 2011)

100. Pagida salticiformis (Pickard-Cambridge, 1883)

- Tamil Nadu (Caleb, 2020a)

Elsewhere: Sri Lanka

101. Pasias marathas Tikader, 1965*

- Maharashtra (Tikader, 1965, 1971, 1980; Pande et al., 2013)

102. Pasias puspagiri Tikader, 1963*

- Karnataka (Tikader, 1963, 1971, 1980)

103. Pharta indica Sen, Saha \& Raychaudhuri, 2012*

- West Bengal (Sen et al., 2012, 2015)
104. Phrynarachne ceylonica (O. Pickard-Cambridge, 1884)

- Andaman \& Nicobar Islands (Dash \& Sivaperuman, 2021b)

- Assam (Das et al., 2019)

Elsewhere: China, Japan, Sri Lanka, Taiwan

105. Phrynarachne peeliana (Stoliczka, 1869)*

Syn. Thomisus peelianus Stoliczka, 1869

- Assam (Stoliczka, 1869)

- West Bengal (Roy et al., 2010)

106. Phrynarachne tuberosa (Blackwall, 1864)*

Syn. Thomisus tuberosus Blackwall, 1864

- West Bengal (Roy et al., 2010)

107. Phrynarachne sp.

- Assam (Das et al., 2015; Pandit, 2019)

108. Pistius barchensis Basu, 1965*

- Uttarakhand (Basu, 1965a; Tikader, 1971, 1980)

- West Bengal (Saha \& Raychaudhuri, 2007a)

109. Pistius bhadurii Basu, 1965*

- Uttar Pradesh (Basu, 1965a; Tikader, 1971)

- Uttarakhand (Gupta \& Siliwal, 2012)

110. Pistius gangulyi Basu, 1965

- Uttarakhand (Basu, 1965a; Tikader, 1971, 1980)

- West Bengal (Saha \& Raychaudhuri, 2007a)

Elsewhere: China

111. Pistius kalimpus Tikader, 1970*

- Maharashtra (Warghat et al., 2014)

- Sikkim (Tikader, 1970)

- West Bengal (Tikader, 1970, 1971, 1980; Saha \& Raychaudhuri, 2007a)

112. Pistius kanikae Basu, 1964*

- Uttarakhand (Basu, 1964a; Tikader, 1971, 1980)

113. Pistius robustus Basu, 1965*

- Maharashtra (Meshram, 2011)

- Uttarakhand (Basu, 1965a; Tikader, 1971, 1980)

114. Pistius tikaderi Kumari \& Mittal, 1999*

- Punjab (Kumari \& Mittal, 1999)

115. Pistius sp.

- Kerala (Sudhikumar et al., 2005; Sunil Jose et al., 2008)

116. Platythomisus sudeepi Biswas, 1977

- Goa (Bastawade \& Borkar, 2008)

- Karnataka (Biswas, 1977; Tikader, 1980; Yadav et al., 2017a)

- Kerala (Siliwal \& Molur, 2005)

- Maharashtra (Yadav et al., 2017b)

Elsewhere: Sri Lanka

117. Platythomisus xiandao Lin \& Li, 2019

Syn. Platythomisus octomaculatus (C. L. Koch, 1845)

- Assam (Yadav et al., 2017b) 
Elsewhere: China

118. Psammitis minor (Charitonov, 1946)

Syn. Xysticus minor Charitonov, 1946).

- Kerala (Sumesh \& Sudhikumar, 2020)

Elsewhere: Central Asia, Iran, Kazakistan

119. Psammitis setiger (O. Pickard-Cambridge, 1885)

Syn. Xysticus setiger O. Pickard-Cambridge, 1885

- Jammu \& Kashmir (Caporiacco, 1935)

Elsewhere: Pakistan

120. Psammitis xysticiformis (Caporiacco, 1935)

Syn. Oxyptila xysticiformis Caporiacco, 1935)

- Jammu \& Kashmir (Caporiacco, 1935)

Elsewhere: Central Asia, China, Pakistan

\section{Runcinia acuminata (Thorell, 1881)}

- Karnataka (Nalini Bai \& Ravindranatha, 2012)

- Maharashtra (Chapke, 2012)

- Manipur (Kananbala et al., 2018)

Elsewhere: Australia, Bangladesh, Japan, New Guinea

122. Runcinia bifrons (Simon, 1895)

Syn. Runciniopsis bifrons Simon, 1895

- Sikkim (Simon, 1895b)

Elsewhere: Sri Lanka, Vietnam

123. Runcinia escheri Reimoser, 1934*

- Andhra Pradesh (Tikader, 1971, 1980; Gajbe, 2007)

- Madhya Pradesh (Gajbe, 2003b, 2005)

- Tamil Nadu (Reimoser, 1934; Karthikeyani et al., 2017)

124. Runcinia ghorpadei Tikader, 1980*

- Gujarat (Siliwal et al., 2003a; Yadav et al., 2017a).

- Karnataka (Tikader, 1980).

- Maharashtra (More \& Sawant, 2013).

125. Runcinia insecta (L. Koch, 1875)

Syn. Runcinia affinis Simon, 1897; Runcinia albostriata Bösenberg \& Strand, 1906; Runcinia chauhani Sen \& Basu, 1973; Thomisus cherapunjeus Tikader, 1966.

- Andaman \& Nicobar Islands (Tikader, 1977).

- Arunachal Pradesh (Biswas \& Biswas, 2006).

- Assam (Tikader, 1966a; Tikader \& Biswas, 1981).

- Chhattisgarh (Gajbe, 2003b; Gajbe \& Sharma, 1994; Kujur \& Ekka, 2016).

- Gujarat (Patel \& Pillai, 1988; Siliwal et al., 2003a; Parasharya \& Pathan, 2013; Chandra et al., 2021).

- Haryana (Malik \& Goyal, 2017).

- Jammu \& Kashmir (Khan, 2009).

- Karnataka (Kokilamani et al., 2019; Sharma \& Ramakrishna, 2021).

- Kerala (Jose et al., 2018)

- Madhya Pradesh (Gajbe, 1995a, b, , 2003B, 2007)

- Maharashtra (Maheshwari et al., 2018)

- Manipur (Kananbala et al., 2018)
- Meghalaya (Tikader, 1966a, 1971, 1980; Tikader \& Biswas, 1981; Biswas \& Majumder, 1995; Majumder, 2005; ; Gajbe, 2007; Chapke, 2012)

- Tamil Nadu (Ganesh Kumar \& Velusamy, 1996; Karthikeyani et al., 2017; Caleb \& Karthikeyani, 2020; Caleb, 2020a)

- Uttar Pradesh (Hore \& Uniyal, 2008a, b; Uniyal \& Hore, 2009)

- Uttarakhand (Gupta \& Siliwal, 2012)

- West Bengal (Tikader, 1977; Tikader \& Biswas, 1981; Biswas \& Biswas, 1992; Majumder, 2005; Saha et al., 2020)

Elsewhere: Africa, Asia, Australia

126. Runcinia khandari Gajbe, 2004*

- Chhattisgarh (Ekka \& Kujur, 2015; Kujur \& Ekka, 2016)

- Madhya Pradesh (Gajbe, 2004d; Patil, 2012)

- Maharashtra (Rithe, 2012; More \& Sawant, 2013;

Warghat et al., 2014)

127. Runcinia kinbergi Thorell, 1891

- Andaman \& Nicobar Islands (Thorell, 1891)

Elsewhere: Indonesia, Myanmar

128. Runcinia roonwali Tikader, 1965*

- Assam (Tikader, 1968)

- Gujarat (Yadav et al., 2017a).

- Haryana (Malik \& Goyal, 2017).

- Karnataka (Deshpande \& Paul, 2016).

- Kerala (Joseph \& Premila, 2016; Adarsh \& Nameer, 2016; Jose et al., 2018; Sumesh \& Sudhikumar, 2020).

- Maharashtra (Tikader, 1965, 1971, 1980).

- Meghalaya (Tikader, 1971, 1980; Biswas \& Majumder, 1995).

- Uttar Pradesh (Uniyal \& Hore, 2009).

129. Runcinia sitadongri Gajbe, 2004*

- Madhya Pradesh (Gajbe, 2004a).

130. Runcinia yogeshi Gajbe \& Gajbe, 2001*

- Chhattisgarh (Ekka \& Kujur, 2015; Kujur \& Ekka, 2016).

- Madhya Pradesh (Gajbe \& Gajbe, 2000a; Gajbe, 2003a, b; Patil, 2012).

- Maharashtra (More \& Sawant, 2013).

131. Runcinia sp.

- Gujarat (Parmar et al., 2015)

- Jammu \& Kashmir (Khan, 2009)

- Karnataka (Mubeen \& Basavarajappa, 2018)

- Kerala (Mathew et al., 2014)

- Madhya Pradesh (Gajbe, 2004c)

- Maharashtra (Nerlekar et al., 2016; Lanka et al., 2017)

- Odisha (Mohapatra et al., 2014)

- Tamil Nadu (Kapoor, 2008)

- Uttar Pradesh (Hore \& Uniyal, 2008a, b)

- Uttarakhand (Uniyal \& Hore, 2006; Uniyal et al., 2011) 
132. Stiphropus duriusculus (Simon, 1885)*

Syn. Apsectromerus duriusculus Simon, 1885)

- Karnataka (Simon, 1885a)

133. Stiphropus sangayus Barrion \& Litsinger, 1995

- Madhya Pradesh (Sen et al., 2010b)

Elsewhere: Philippines

134. Stiphropus soureni Sen, 1964

- West Bengal (Sen, 1964)

Elsewhere: Bhutan

135. Stiphropus sp.

- Goa (Pandit \& Dharwadkar, 2020)

136. Strigoplus bilobus Saha \& Raychaudhuri, 2004*

- West Bengal (Saha \& Raychaudhuri, 2004)

137. Strigoplus moluri Patel, 2003*

- Kerala (Patel, 2003a)

- Maharashtra (More \& Sawant, 2013)

138. Strigoplus netravati Tikader, 1963*

- Goa (Bastawade \& Borkar, 2008; Pandit \& Dharwadkar, 2020)

- Karnataka (Tikader, 1963, 1971, 1980)

- Kerala (Sunil Jose et al., 2008; Asima et al., 2020; Sumesh \& Sudhikumar, 2020; Smitha \& Sudhikumar, 2020)

- Maharashtra (Bastawade \& Sharma, 2002)

139. Strigoplus sp.

- Goa (Pandit \& Pai, 2017)

- Karnataka (Murali et al., 2017)

- Kerala (Joseph \& Premila, 2016)

- Maharashtra (Warghat et al., 2011)

140. Synema decoratum Tikader, 1960.

Syn. Synaema decorata Tikader, 1960

- Chhattisgarh (Gajbe, 2003b, 2004d, 2005; Kujur \& Ekka, 2016)

- Gujarat (Parmar, 2013; Parmar \& Acharya, 2015; Parmar \& Patel, 2017; Parmar et al., 2015; Yadav et al., 2017a)

- Karnataka (Tikader, 1971, 1980; Tikader \& Biswas, 1981; Gajbe, 2004d, 2007; Majumder, 2005)

- Kerala (Adarsh \& Nameer, 2016)

- Madhya Pradesh (Gajbe, 2003a, b, 2004d, 2005, 2007; Patil, 2012)

- Maharashtra (Tikader, 1971, 1980; Gajbe, 2004d, 2007; Majumder, 2005; Majumder, 2005; Vairale \& Wagh, 2021)

- Tamil Nadu (Caleb, 2020a)

- Uttarakhand (Uniyal et al., 2011)

- West Bengal (Tikader, 1960, 1971, 1980; Tikader \& Biswas, 1981; Biswas \& Biswas, 1992; Gajbe, 2004d, 2007)

Elsewhere: China

141. Synema mysorense Tikader, 1980*
Syn. Synaema mysorensis Tikader, 1980

- Chhattisgarh (Kujur \& Ekka, 2016)

- Karnataka (Tikader, 1980; Gajbe, 2007)

- Madhya Pradesh (Gajbe, 2003b, 2007)

142. Synema revolutum Tang \& Li, 2010

- Goa (Pandit \& Dharwadkar, 2020)

- West Bengal (Sen et al., 2012, 2015)

Elsewhere: China

143. Synema sp.

- Goa (Pandit \& Pai, 2017)

- Rajasthan (Sivaperuman \& Rathore, 2004)

144. Talaus opportunus (Pickard-Cambridge, 1873)

- Kerala (Adarsh \& Nameer, 2016)

- Tamil Nadu (Simon, 1906)

Elsewhere: Sri Lanka

145. Tharpyna himachalensis Tikader \& Biswas, 1979*

- Himachal Pradesh (Tikader \& Biswas, 1979)

146. Tharpyna indica Tikader \& Biswas, 1979*

- West Bengal (Tikader \& Biswas, 1979, 1981)

147. Tharpyna sp.

- Jharkhand (Agrawal \& Ghosh, 1995)

148. Tharrhalea evanida (L. Koch, 1867).

Syn. Diaea evanida L. Koch, 1867

- Haryana (Malik \& Goyal, 2017)

- Maharashtra (Warghat et al., 2011)

Elsewhere: Australia, New Guinea

149. Thomisus andamanensis Tikader, 1980*

- Andaman \& Nicobar Islands (Tikader, 1980)

- Delhi (Malik et al., 2015)

- Gujarat (Trivedi, 2009)

- Karnataka (Nautiyal et al., 2017)

- Kerala (Sebastian et al., 2005b, 2011; Jose et al., 2007; Dhali et al., 2019)

- Maharashtra (Warghat et al., 2011, 2014)

- Meghalaya (Roy et al., 2017)

- Rajasthan (Sen et al., 2009; Saha et al., 2015b)

- West Bengal (Sen et al., 2015; Raychaudhuri et al., 2016;

Saha et al., 2016; Chandra et al., 2021)

150. Thomisus armillatus (Thorell, 1891)*

Syn. Daradius armillatus Thorell, 1891

- Andaman \& Nicobar Islands (Thorell, 1891; Leardi in Airaghi, 1901b).

151. Thomisus ashishi Gajbe, 2005*

- Madhya Pradesh (Gajbe, 2005).

- Maharashtra (Rithe, 2012).

152. Thomisus baghdeoi Gajbe, 2004*

- Madhya Pradesh (Gajbe, 2004a)

153. Thomisus bargi Gajbe, 2004* 
- Chhattisgarh (Ekka \& Kujur, 2015; Kujur \& Ekka, 2016)

- Madhya Pradesh (Gajbe, 2004d; Patil, 2012).

154. Thomisus beautifularis Basu, 1965*

- Maharashtra (Deshmukh \& Tekade, 2019; Vairale \& Wagh, 2021).

- Tamil Nadu (Ganesh Kumar \& Velusamy, 1996; Sugimaran et al., 2007).

- West Bengal (Basu, 1965b; Biswas \& Biswas, 1992; Majumder, 2005).

155. Thomisus bulani Tikader, 1960*

- Gujarat (Siliwal et al., 2003b; Patel, 2003b)

- West Bengal (Tikader, 1960, 1971, 1980; Tikader \& Biswas, 1981; Biswas \& Biswas, 1992; Majumder, 2005; Saha \& Raychaudhuri, 2007a).

156. Thomisus danieli Gajbe, 2004*

- Chhattisgarh (Ekka \& Kujur, 2015; Kujur \& Ekka, 2016).

- Madhya Pradesh (Gajbe, 2004d; Patil, 2012)

- Maharashtra (Warghat et al., 2014)

157. Thomisus daradioides Simon, 1890

- India (Bonnet, 1959)

Elsewhere: Pakistan, South Africa, Sudan, Yemen

158. Thomisus dentiger (Thorell, 1887)

Syn. Daradius dentiger Thorell, 1887)

- Uttarakhand (Leardi in Airaghi, 1901a)

Elsewhere: Myanmar

159. Thomisus dhakuriensis Tikader, 1960*

- Gujarat (Kumar \& Shivakumar, 2006; Yadav, 2019)

- West Bengal (Tikader, 1960, 1971, 1980; Tikader \& Biswas, 1981; Biswas \& Biswas, 1992; Majumder, 2005)

160. Thomisus dhananjayi Gajbe, 2005*

- Madhya Pradesh (Gajbe, 2005)

161. Thomisus dyali Kumari \& Mittal, 1997*

- Punjab (Kumari \& Mittal, 1997)

162. Thomisus elongatus Stoliczka, 1869*

- Chhattisgarh (Gajbe, 2003b).

- Gujarat (Patel \& Pillai, 1988; Siliwal et al., 2003a).

- Jammu \& Kashmir (Khan, 2006).

- Madhya Pradesh (Gajbe, 2007).

- Maharashtra (Meshram, 2011; Vairale \& Wagh, 2021).

- West Bengal (Stoliczka, 1869; Tikader, 1971, 1980; Tikader \& Biswas, 1981; Biswas \& Biswas, 1992; Majumder, 2005; Gajbe, 2007).

163. Thomisus godavariae Reddy \& Patel, 1992*

- Andhra Pradesh (Reddy \& Patel, 1992)

164. Thomisus granulifrons Simon, 1906.

- Maharashtra (More \& Sawant, 2013; More, 2015a).

- Puducherry (Simon, 1906).

- Tamil Nadu (Simon, 1906; Karthikeyani et al., 2017).

Elsewhere: Sri Lanka
165. Thomisus italongus Barrion \& Litsinger, 1995

- Rajasthan (Sen et al., 2009; Saha et al., 2015b)

Elsewhere: Philippines.

166. Thomisus katrajghatus Tikader, 1963*

- Madhya Pradesh (Tikader, 1971; Gajbe, 2003b, 2007)

- Maharashtra (Tikader, 1963, 1980; Tikader \& Biswas, 1981; Gajbe, 2007; Pande et al., 2013).

- West Bengal (Tikader \& Biswas, 1981; Biswas \& Biswas, 1992; Majumder, 2005)

167. Thomisus keralae Biswas \& Roy, 2005*

- Kerala (Biswas \& Roy, 2005a)

168. Thomisus kokiwadai Gajbe, 2004*

- Madhya Pradesh (Gajbe, 2004a)

169. Thomisus krishnae Reddy \& Patel, 1992*

- Andhra Pradesh (Reddy \& Patel, 1992)

- Gujarat (Patel, 2003b; Siliwal et al., 2003b; Kumar \& Yashkamal, 2011)

170. Thomisus laglaizei Simon, 1877

- Tamil Nadu (Leardi in Airaghi, 1901b)

Elsewhere: Indonesia, Myanmar, Philippines

171. Thomisus leucaspis Simon, 1906

- Tamil Nadu (Simon, 1906; Karthikeyani et al., 2017)

Elsewhere: New Caledonia

172. Thomisus lobosus Tikader, 1965*

- Assam (Chetia \& Kalita, 2012; Basumatary \& Brahma, 2017)

- Chhattisgarh (Gajbe, 2003b, 2005; Kujur \& Ekka, 2016)

- Gujarat (Parmar, 2013; Parmar \& Patel, 2017; Parmar et al., 2015)

- Jammu \& Kashmir (Chandra et al., 2021)

- Karnataka (Nalini Bai \& Ravindranatha, 2012; Bhat et al., 2013; Vaibhav et al., 2017)

- Kerala (Sunil Jose \& Sebastian, 2001; Sebastian et al., 2005a; Sebastian et al., 2011; Jose et al., 2018; Rajeevan et al., 2019)

- Madhya Pradesh (Gajbe, 2007)

- Maharashtra (Tikader, 1965, 1980; Gajbe, 2005, 2007; Lanka et al., 2017)

- Odisha (Mohapatra et al., 2014)

- Rajasthan (Kaur et al., 2014; Lawania \& Trigunayat, 2015; Kashmeera et al., 2020)

- Tamil Nadu (Karthikeyani et al., 2017; Kadam \& Rajkumar, 2020)

- Uttar Pradesh (Lawania \& Mathur, 2014a, b, c, d)

- Uttarakhand (Gupta \& Siliwal, 2012; Pooja et al., 2019)

173. Thomisus ludhianaensis Kumari \& Mittal, 1997*

- Punjab (Kumari \& Mittal, 1997)

174. Thomisus manishae Gajbe, 2005*

- Chhattisgarh (Kujur \& Ekka, 2012; Ekka \& Kujur, 2015)

- Madhya Pradesh (Gajbe, 2005) 
175. Thomisus manjuae Gajbe, 2005*

- Madhya Pradesh (Gajbe, 2005)

176. Thomisus meenae Gajbe, 2005*

- Madhya Pradesh (Gajbe, 2005)

177. Thomisus mimae Sen \& Basu, 1963*

- Maharashtra (Vairale \& Wagh, 2021)

- West Bengal (Sen \& Basu, 1963; Tikader, 1971, 1980;

Biswas \& Biswas, 1992; Majumder, 2005)

178. Thomisus nirmali Saha \& Raychaudhuri, 2007*

- Jammu \& Kashmir (Punjoo \& Bhat, 2015)

- West Bengal (Saha \& Raychaudhuri, 2007b)

179. Thomisus okinawensis Strand, 1907

- Madhya Pradesh (Keswani, 2014)

- Maharashtra (Maheshwari et al., 2018)

Elsewhere: Indonesia, Japan, Philippines, Thailand

180. Thomisus onustus Walckenaer, 1805

Syn. Thomisus diadema Hahn, 1832

- Andaman \& Nicobar Islands (Thorell, 1891)

- Uttarakhand (Uniyal et al., 2011)

Elsewhere: Africa, Belgium, Britain, Caucasus, China, Europe, France, Germany, Iran, Israel, Japan, Korea, Mediterranean, North Africa, Palestine, Poland, Russia, Selvagens Is., Sweden, Turkey, Yemen

\section{Thomisus pateli Gajbe, 2004*}

- Chhattisgarh (Kujur \& Ekka, 2012; Ekka \& Kujur, 2015)

- Madhya Pradesh (Gajbe, 2004d; Patil, 2012)

- Maharashtra (Warghat et al., 2014)

182. Thomisus pathaki Gajbe, 2004 *

- Maharashtra (More \& Sawant, 2013; More, 2015a, b)

- Chhattisgarh (Ekka \& Kujur, 2015)

- Madhya Pradesh (Gajbe, 2004d; Patil, 2012; )

183. Thomisus pooneus Tikader, 1965*

- Haryana (Malik \& Goyal, 2017)

- Madhya Pradesh (Patil et al., 2013)

- Maharashtra (Tikader, 1965, 1980; More \& Sawant, 2013; Pande et al., 2013; Vairale \& Wagh, 2021)

- Uttar Pradesh (Chandra et al., 2021)

184. Thomisus pritiae Gajbe, 2005*

- Gujarat (Parmar \& Patel, 2017)

- Madhya Pradesh (Gajbe, 2005)

185. Thomisus projectus Tikader, 1960*

- Assam (Chetia \& Kalita, 2012)

- Chhattisgarh (Gajbe, 2003b; Gajbe \& Sharma, 1994; Kujur \& Ekka, 2016)

- Gujarat (Patel, 2003b; Trivedi, 2009; Parmar, 2013; Parasharya \& Pathan, 2013; Parmar et al., 2015; Yadav et al., 2017a)

- Haryana (Malik \& Goyal, 2017)

- Jammu \& Kashmir (Chandra et al., 2021)
- Karnataka (Murali et al., 2017)

- Kerala (Sebastian et al., 2011; Ambily \& Antony, 2016; Jose et al., 2018; Rajeevan et al., 2019; Sumesh \& Sudhikumar, 2020)

- Madhya Pradesh (Tikader, 1971; Gajbe, 1995b, 2003B, 2007)

- Maharashtra (Warghat et al., 2011; Vairale \& Wagh, 2021)

- Manipur (Biswas \& Biswas, 2004)

- Odisha (Panda et al., 2011)

- Rajasthan (Chauhan et al., 2009; Lawania \& Trigunayat, 2015; Kumari et al., 2017)

- Telangana (Pravalikha \& Srinivasulu, 2015)

- Uttar Pradesh (Lawania \& Mathur, 2014b, c, d)

- West Bengal (Tikader, 1960, 1980; Tikader \& Biswas, 1981; Biswas \& Biswas, 1992; Majumder, 2005; Gajbe, 2007)

186. Thomisus pugilis Stoliczka, 1869*

- Assam (Gupta et al., 2015)

- Gujarat (Patel \& Pillai, 1988; Siliwal et al., 2003a; Prajapati et al., 2016)

- Jammu \& Kashmir (Khan, 2006)

- Karnataka (Nalini Bai \& Ravindranatha, 2012; Nautiyal et al., 2017; Kokilamani et al., 2019)

- Kerala (Sebastian et al., 2005a; Mathew et al., 2014; Asima et al., 2020; Smitha \& Sudhikumar, 2020)

- Lakshadweep (Pocock, 1904)

- Madhya Pradesh (Tikader, 1971; Gajbe, 1995a, 2003b; Patil, 2012)

- Maharashtra (Lanka et al., 2017; Vairale \& Wagh, 2021)

- Mizoram (Chowdhury et al., 2017)

- Odisha (Panda et al., 2011)

- Punjab (Tikader, 1971, 1980; Tikader \& Biswas, 1981; Majumder, 2005; Caleb, 2020a)

- Rajasthan (Kaur et al., 2014)

- Tamil Nadu (Leardi in Airaghi, 1901b; Tikader, 1971, 1980; Tikader \& Biswas, 1981; Ganesh Kumar \& Velusamy, 1996; Majumder, 2005; Gajbe, 2005; Karthikeyani et al., 2017; Caleb, 2020a).

- Uttar Pradesh (Hore \& Uniyal, 2008a, b; Uniyal \& Hore, 2009)

- West Bengal (Stoliczka, 1869; Leardi in Airaghi, 1901b; Tikader, 1980; Tikader \& Biswas, 1981; Biswas \& Biswas, 1992; Majumder, 2005; Caleb, 2020a)

187. Thomisus rajani Bhandari \& Gajbe, 2001*

- Chhattisgarh (Kujur \& Ekka, 2012; Ekka \& Kujur, 2015; Kujur \& Ekka, 2016)

- Madhya Pradesh (Bhandari \& Gajbe, 2001; Gajbe, 2003a, b, 2004d; Patil, 2012)

- Maharashtra (Rithe, 2012)

188. Thomisus rigoratus Simon, 1906* 
- Tamil Nadu (Simon, 1906; Leardi in Airaghi, 1901b; Karthikeyani et al., 2017)

189. Thomisus rishus Tikader, 1970*

- Sikkim (Tikader, 1970, 1971, 1980)

190. Thomisus shillongensis Sen, 1963*

- Meghalaya (Sen, 1963; Tikader, 1968, 1971, 1980; Biswas \& Majumder, 1995)

191. Thomisus shivajiensis Tikader, 1965*

- Gujarat (Siliwal et al., 2003b; Solanki \& Kumar, 2014)

- Haryana (Malik \& Goyal, 2017)

- Maharashtra (Tikader, 1965, 1971, 1980; Meshram, 2011)

- Punjab (Kumari, 1983)

192. Thomisus sikkimensis Tikader, 1962*

- Odisha (Chetry \& Moran, 2019)

- Sikkim (Tikader, 1962, 1970, 1980)

- West Bengal (Saha et al., 2020)

193. Thomisus simoni Gajbe, 2004*

- Madhya Pradesh (Gajbe, 2004d; Patil, 2012)

- Chhattisgarh (Ekka \& Kujur, 2015; Kujur \& Ekka, 2016)

194. Thomisus sorajaii Basu, 1963*

- Maharashtra (Bastawade, 2008).

- Uttar Pradesh (Chandra et al., 2021).

- West Bengal (Basu, 1963; Tikader, 1971, 1980; Biswas \& Biswas, 1992; Majumder, 2005).

195. Thomisus spectabilis Doleschall, 1859

Syn. Daradius annulipes Thorell, 1877.

- Goa (Pandit \& Pai, 2017).

- Gujarat (ICAR-NBAIR, 2019).

- Karnataka (Shraddha \& Chaturved, 2019, 2020).

- Kerala (Vineetha \& George, 2021).

- Maharashtra (Warghat et al., 2011)

- Puducherry (Leardi in Airaghi, 1901b)

- Tamil Nadu (Reimoser, 1934)

- West Bengal (Ghosh et al., 2018)

Elsewhere: Australia, Indonesia, Malaysia, Micronesia, New Guinea,

196. Thomisus stoliczkai (Thorell, 1887)

- Karnataka (Leardi in Airaghi, 1901b)

Elsewhere: Myanmar

197. Thomisus sundari Gajbe \& Gajbe, 2000*

- Chhattisgarh (Kujur \& Ekka, 2016)

- Madhya Pradesh (Gajbe \& Gajbe, 2000b; Gajbe, 2003a, b; Patil, 2012)

198. Thomisus telanganaensis Pravalikha \& Srinivasulu, 2015*

- Kerala (Rajeevan et al., 2019)

- Telangana (Pravalikha \& Srinivasulu, 2015)
199. Thomisus unidentatus Dippenaar-Schoeman \& van Harten, 2007

- Delhi (Diksha et al., 2018)

- Uttar Pradesh (Diksha et al., 2018)

Elsewhere: Iran, Iraq, Yemen

200. Thomisus viveki Gajbe, 2004*

- Chhattisgarh (Kujur \& Ekka, 2012; Ekka \& Kujur, 2015)

- Madhya Pradesh (Gajbe, 2004d; Patil, 2012)

- Maharashtra (Rithe, 2012; More \& Sawant, 2013;

Warghat et al., 2014)

201. Thomisus whitakeri Gajbe, 2004*

- Chhattisgarh (Ekka \& Kujur, 2015)

- Madhya Pradesh (Gajbe, 2004d; Patil, 2012)

- Maharashtra (Meshram, 2011; Vairale \& Wagh, 2021)

202. Thomisus sp.

- Andhra Pradesh (Palem et al., 2016)

- Assam (Das et al., 2015; Pandit, 2019)

- Bihar (Yadav et al., 2016)

- Chhattisgarh (Ekka \& Kujur, 2015)

- Goa (Pandit \& Dharwadkar, 2020).

- Gujarat (Siliwal et al., 2003a; Yadav et al., 2017a).

- Jammu \& Kashmir (Khan, 2009; Khan \& Rather, 2012).

- Kerala (Sebastian et al., 2005b)

- Madhya Pradesh (Gajbe, 2004b; Sen et al., 2010b).

- Maharashtra (Nerlekar et al., 2016)

- Odisha (Choudhury et al., 2019)

- Tamil Nadu (Kapoor, 2008; Caleb, 2020b)

- Uttar Pradesh (Khan \& Mishra, 2003; Chandra et al., 2021)

- Uttarakhand (Uniyal \& Hore, 2006; Pooja et al., 2019)

203. Tmarus dejectus (O. Pickard-Cambridge, 1885)*

Syn. Monastes dejectus $\boldsymbol{O}$. Pickard-Cambridge, 1885

- Jammu \& Kashmir (Pickard-Cambridge, 1885; Caporiacco, 1935)

Elsewhere: Pakistan

204. Tmarus fasciolatus Simon, 1906*

- Puducherry (Simon, 1906)

- Tamil Nadu (Simon, 1906; Leardi in Airaghi, 1901b; Karthikeyani et al., 2017)

205. Tmarus jabalpurensis Gajbe \& Gajbe, 1999*

- Chhattisgarh (Kujur \& Ekka, 2012; Ekka \& Kujur, 2015)

- Madhya Pradesh (Gajbe \& Gajbe, 1999a; Gajbe, 2003a, b, 2004d; Patil, 2012)

- Maharashtra (Rithe, 2012; More \& Sawant, 2013; More, 2015a)

206. Tmarus kotigeharus Tikader, 1963*

- Assam (Tikader, 1968)

- Gujarat (Patel, 2003b; Yadav et al., 2017a)

- Karnataka (Tikader, 1963, 1971, 1980) 
- Kerala (Sumesh \& Sudhikumar, 2020)

- Maharashtra (Tikader, 1971; Bastawade \& Khandal, 2006; More \& Sawant, 2013; More, 2015a)

- Meghalaya (Tikader, 1971)

- Punjab (Kumari, 1983)

- Rajasthan (Kashmeera et al., 2020)

- West Bengal (Sen et al., 2015)

207. Tmarus soricinus Simon, 1906*

- Kerala (Joseph et al., 2017)

- Tamil Nadu (Simon, 1906; Leardi in Airaghi, 1901b; Karthikeyani et al., 2017)

208. Tmarus srisailamensis Rao et al., 2006*

- Andhra Pradesh (Rao et al., 2006)

209. Tmarus stellio Simon, 1875

- Jammu \& Kashmir (Caporiacco, 1935)

Elsewhere: Caucasus, Europe, Iran, Turkey.

210. Tmarus sp.

- Assam (Basumatary \& Brahma, 2017)

- Gujarat (Siliwal et al., 2003a)

- Madhya Pradesh (Gajbe, 2004b)

- Manipur (Kananbala et al., 2018)

- Rajasthan (Chauhan et al., 2009; Kumari et al., 2017; Lawania \& Mathur, 2017)

- Tamil Nadu (Kapoor, 2008; Kadam \& Rajkumar, 2020)

211. Xysticus audax (Schrank, 1803)

Syn. Xysticus pini (Hahn, 1931)

- Jammu \& Kashmir (Caporiacco, 1935)

- Kerala (Sumesh \& Sudhikumar, 2020)

Elsewhere: Caucasus, Europe, Iran, Japan, Kazakhstan, Korea, Russia, Turkey

212. Xysticus bengalensis Tikader \& Biswas, 1974*

- Madhya Pradesh (Gajbe, 2003a, b, 2004d, 2007; Patil, 2012)

- Rajasthan (Malhotra et al., 2019)

- West Bengal (Tikader \& Biswas, 1974; Tikader, 1980; Biswas \& Biswas, 1992; Majumder, 2005)

213. Xysticus bengdakus Saha \& Raychaudhuri, 2007*

- West Bengal (Saha \& Raychaudhuri, 2007a)

214. Xysticus bharatae Gajbe \& Gajbe, 1999*

- Chhattisgarh (Ekka \& Kujur, 2015)

- Madhya Pradesh (Gajbe \& Gajbe, 1999b; Gajbe, 2003a, b, 2004d; Patil, 2012)

- Maharashtra (Rithe, 2012; More, 2015a, b)

215. Xysticus breviceps O. Pickard-Cambridge, 1885*

- Gujarat (Parmar, 2013; Parmar et al., 2015)

- Kerala (Adarsh \& Nameer, 2016; Joseph et al., 2017; Asima et al., 2020)

- Ladakh (Pickard-Cambridge, 1885)

- Maharashtra (Rithe, 2012; More \& Sawant, 2013; More, 2015a).
216. Xysticus cristatus (Clerck, 1757) .

Syn. Daradius cristatus (Clerck, 1757).

- Jammu \& Kashmir (Caporiacco, 1935; Punjoo \& Bhat, 2015).

- Kerala (Sumesh \& Sudhikumar, 2020).

- Puducherry (Leardi in Airaghi, 1901b).

Elsewhere: Canada, Caucasus, Central Asia, China, Europe, Iran, Japan, Kazakhstan, Korea, Russia, Turkey, USA.

\section{Xysticus croceus Fox, 1937}

Syn. Xysticus sujatai Tikader, 1962)

- Arunachal Pradesh (Tikader, 1971; Biswas \& Biswas, 2006)

- Gujarat (Patel \& Pillai, 1988)

- Meghalaya (Tikader, 1962, 1971, 1980; Biswas \& Majumder, 1995)

- Mizoram (Biswas \& Biswas, 2007)

- Uttarakhand (Quasin \& Uniyal, 2010; Uniyal et al., 2011)

- West Bengal (Biswas \& Biswas, 2006)

Elsewhere: Bhutan, China, Japan, Korea, Nepal, Taiwan, Vietnam

218. Xysticus himalayaensis Tikader \& Biswas, 1974*

- Kerala (Sudhikumar et al., 2005; Sunil Jose et al., 2008)

- West Bengal (Tikader \& Biswas, 1974; Tikader, 1980; Biswas \& Biswas, 1992)

219. Xysticus hindusthanicus Basu, 1965*

- West Bengal (Basu, 1965b; Tikader, 1971, 1980; Biswas \& Biswas, 1992; Majumder, 2005)

220. Xysticus jabalpurensis Gajbe \& Gajbe, 1999*

- Chhattisgarh (Ekka \& Kujur, 2015; Kujur \& Ekka, 2016)

- Madhya Pradesh (Gajbe \& Gajbe, 1999b; Gajbe, 2003a, b, 2004d; Patil, 2012)

- Maharashtra (Rithe, 2012)

221. Xysticus jaharai Basu, 1979*

- Uttarakhand (Basu, 1979)

222. Xysticus joyantius Tikader, 1966*

Syn. Xysticus jayantius Tikader, 1966)

- Chhattisgarh (Gajbe, 2003b; Ekka \& Kujur, 2015; Kujur \& Ekka, 2016)

- Gujarat (Parmar, 2013)

- Madhya Pradesh (Gajbe, 2003b, 2007)

- Maharashtra (Meshram, 2011; Vairale \& Wagh, 2021)

- Meghalaya (Tikader, 1966b, 1968, 1971, 1980; Biswas \& Majumder, 1995; Gajbe, 2007).

- Uttarakhand (Uniyal et al., 2011).

223. Xysticus kali Tikader \& Biswas, 1974*

- Chhattisgarh (Ekka \& Kujur, 2015; Kujur \& Ekka, 2016)

- Madhya Pradesh (Gajbe, 2003b, 2005, 2007).

- Maharashtra (Meshram, 2011).

- Uttarakhand (Uniyal et al., 2011) 
- West Bengal (Tikader \& Biswas, 1974; Tikader, 1980; Biswas \& Biswas, 1992; Gajbe, 2005)

224. Xysticus kamakhyai Tikader, 1962*

- Meghalaya (Tikader, 1962, 1971, 1980; Biswas \& Majumder, 1995)

225. Xysticus kashidi Tikader, 1963*

- Karnataka (Tikader, 1963, 1971, 1980)

226. Xysticus khasiensis Tikader, 1980*

- Chhattisgarh (Gajbe \& Sharma, 1994)

- Maharashtra (More \& Sawant, 2013; More, 2015a)

- Meghalaya (Tikader, 1980; Biswas \& Majumder, 1995)

227. Xysticus maculiger Roewer, 1951

Syn. Xysticus maculosus Pickard-Cambridge, 1885)

- Jammu \& Kashmir (Caporiacco, 1935)

Elsewhere: China

228. Xysticus mundulus O. Pickard-Cambridge, 1885*

- Jammu \& Kashmir (O. Pickard-Cambridge, 1885;

Caporiacco, 1935)

229. Xysticus pynurus Tikader, 1966*

- Meghalaya (Tikader, 1966b, 1968, 1971, 1980; Biswas \& Majumder, 1995)

230. Xysticus roonwali Tikader, 1964

- Sikkim (Tikader, 1971, 1980)

Elsewhere: Nepal

231. Xysticus shillongensis Tikader, 1962*

- Meghalaya (Tikader, 1962, 1971, 1980; Biswas \& Majumder, 1995)

\section{Xysticus shyamrupus Tikader, 1966*}

- Madhya Pradesh (Gajbe, 2003b, 2007).

- Meghalaya (Tikader, 1966a, 1971, 1980; Biswas \& Majumder, 1995; Gajbe, 2007).

- Uttarakhand (Biswas \& Biswas, 2010).

233. Xysticus sikkimus Tikader, 1970

- Sikkim (Tikader, 1970, 1971, 1980)

Elsewhere: China

234. Xysticus tikaderi Bhandari \& Gajbe, 2001*

- Chhattisgarh (Kujur \& Ekka, 2012; Ekka \& Kujur, 2015)

- Karnataka (Bhat et al., 2013)

- Madhya Pradesh (Bhandari \& Gajbe, 2001; Gajbe, 2003a, b, 2004a; Patil, 2012)

- Maharashtra (More \& Sawant, 2013; More, 2015a, b).

235. Xysticus viveki Gajbe, 2005*

- Madhya Pradesh (Gajbe, 2005)

236. Xysticus yogeshi Gajbe, 2005*

- Madhya Pradesh (Gajbe, 2005)

237. Xysticus sp.

- Assam (Pandit, 2019)

- Goa (Pandit \& Dharwadkar, 2020)

- Gujarat (Solanki \& Kumar, 2014; Parmar et al., 2015)
- Jammu \& Kashmir (Khan, 2009; Punjoo \& Bhat, 2015)

- Karnataka (Bhat et al., 2013; Nautiyal et al., 2017)

- Kerala (Sebastian et al., 2005a; Sunil Jose et al., 2008)

- Ladakh (Uniyal, 2006)

- Madhya Pradesh (Sen et al., 2010b)

- Maharashtra (Lanka et al., 2017)

- Rajasthan (Malhotra et al., 2019)

- Tamil Nadu (Caleb, 2020b)

- Uttar Pradesh (Anjali \& Prakash, 2012; Kumar et al., 2017a)

- Uttarakhand (Uniyal et al., 2011; Gupta \& Siliwal, 2012).

\section{Checklist of Crab Spiders (Thomisidae) in Indian States}

1. Andhra Pradesh: Camaricus bipunctatus, Runcinia escheri, Thomisus godavariae, Thomisus krishnae, Tmarus srisailamensis

2. Arunachal Pradesh: Camaricus bipunctatus, Camaricus formosus, Runcinia insecta, Xysticus croceus

3. Assam: Amyciaea forticeps, Borboropactus elephantus, Camaricus formosus, Henriksenia hilaris, Indoxysticus minutus, Lysiteles mandali, Misumena vatia, Oxytate elongata, Oxytate virens, Ozyptila manii, Phrynarachne ceylonica, Phrynarachne peeliana, Platythomisus xiandao, Runcinia insecta, Runcinia roonwali, Thomisus lobosus, Thomisus projectus, Thomisus pugilis, Tmarus kotigeharus

4. Bihar: Indoxysticus minutus, Thomisus sp.

5. Chhattisgarh: Amyciaea forticeps, Indoxysticus minutus, Misumenoides gwarighatensis, Monaeses jabalpurensis, Oxytate elongata, Ozyptila amkhasensis, Ozyptila jabalpurensis, Runcinia insecta, Runcinia khandari, Runcinia yogeshi, Synema decoratum, Synema mysorense, Thomisus bargi, Thomisus danieli, Thomisus elongatus, Thomisus lobosus, Thomisus manishae, Thomisus pateli, Thomisus pathaki, Thomisus projectus, Thomisus rajani, Thomisus stoliczkai, Thomisus sundari, Thomisus viveki, Thomisus whitakeri, Tmarus jabalpurensis, Xysticus bharatae, Xysticus jabalpurensis, Xysticus joyantius, Xysticus kali, Xysticus khasiensis, Xysticus tikaderi

6. Goa: Amyciaea forticeps, Angaeus sp., Camaricus formosus, Massuria sp., Misumena vatia, Oxytate sp., Platythomisus sudeepi, Stiphropus sp., Strigoplus netravati, Synema revolutum, Thomisus spectabilis, Xysticus sp.

7. Gujarat: Amyciaea forticeps, Camaricus formosus, Camaricus khandalaensis, Diaea sp., Ebrechtella concinna, Indoxysticus lumbricus, Indoxysticus minutus, Mastira menoka, Misumena sp., Misumenoides sp., Monaeses mukundi, Monaeses parvati, Oxytate elongata, Oxytate virens, Ozyptila maratha, Runcinia ghorpadei, Runcinia insecta, Runcinia roonwali, Synema decoratum, Thomisus andamanensis, Thomisus bulani, Thomisus dhakuriensis, Thomisus elongatus, Thomisus krishnae, Thomisus lobosus, Thomisus pritiae, Thomisus projectus, 
Thomisus pugilis, Thomisus shivajiensis, Thomisus spectabilis, Tmarus kotigeharus, Xysticus breviceps, Xysticus croceus, Xysticus joyantius

8. Haryana: Runcinia insecta, Runcinia roonwali, Tharrhalea evanida, Thomisus pooneus, Thomisus projectus, Thomisus shivajiensis

\section{Himachal Pradesh: Tharpyna himachalensis}

\section{Jharkhand: Tharpyna sp.}

11. Karnataka: Amyciaea forticeps, Angaeus pentagonalis, Bomis sp., Camaricus formosus , Indoxysticus minutus, Loxobates castetsi, Mastira menoka, Misumena annapurna, Misumena indra, Misumena vatia, Misumenoides sp., Misumenops rubrodecoratus, Misumessus oblongus, Oxytate virens, Pasias puspagiri, Platythomisus sudeepi, Runcinia acuminata, Runcinia ghorpadei, Runcinia insecta, Runcinia roonwali, Stiphropus duriusculus, Strigoplus netravati, Synema decoratum, Synema mysorense, Thomisus andamanensis, Thomisus lobosus, Thomisus projectus, Thomisus pugilis, Thomisus spectabilis, Thomisus stoliczkai, Tmarus kotigeharus, Xysticus kashidi, Xysticus tikaderi

12. Kerala: Amyciaea albomaculata, Amyciaea forticeps, Camaricus formosus, Camaricus khandalaensis, Camaricus rinkae, Demogenes andamanensis, Ebrechtella concinna, Epidius armatus, Epidius longipalpis, Epidius parvati, Henriksenia hilaris, Indoxysticus minutus, Mastira menoka, Misumena mridulai, Misumenops sp., Oxytate greenae, Oxytate virens, Ozyptila sp., Pistius sp., Platythomisus sudeepi, Psammitis minor, Runcinia insecta, Runcinia roonwali, Strigoplus moluri, Strigoplus netravati, Synema decoratum, Talaus opportunus, Thomisus andamanensis, Thomisus keralae, Thomisus lobosus, Thomisus projectus, Thomisus pugilis, Thomisus spectabilis, Thomisus telanganaensis, Tmarus kotigeharus, Tmarus soricinus, Xysticus audax, Xysticus breviceps, Xysticus cristatus, Xysticus himalayaensis

13. Madhya Pradesh: Bomis khajuriai, Camaricus formosus, Indoxysticus minutus, Lysiteles brunettii, Misumena ritujae, Misumenoides gwarighatensis, Monaeses jabalpurensis, Monaeses pachpediensis, Oxytate elongata, Oxytate kanishkai, Ozyptila amkhasensis, Ozyptila jabalpurensis, Ozyptila maratha, Runcinia escheri, Runcinia insecta, Runcinia khandari, Runcinia sitadongri, Runcinia yogeshi, Stiphropus sangayus, Synema decoratum, Synema mysorense, Thomisus ashishi, Thomisus baghdeoi, Thomisus bargi, Thomisus danieli, Thomisus dhananjayi, Thomisus elongatus, Thomisus katrajghatus, Thomisus kokiwadai, Thomisus lobosus, Thomisus manishae, Thomisus manjuae, Thomisus meenae, Thomisus okinawensis, Thomisus pateli, Thomisus pathaki, Thomisus pooneus, Thomisus pritiae, Thomisus projectus, Thomisus pugilis, Thomisus rajani, Thomisus stoliczkai, Thomisus sundari, Thomisus viveki, Thomisus whitakeri, Tmarus jabalpurensis, Xysticus bengalensis, Xysticus bharatae, Xysticus jabalpurensis, Xysticus joyantius, Xysticus kali, Xysticus shyamrupus, Xysticus tikaderi, Xysticus viveki, Xysticus yogeshi
14. Maharashtra: Amyciaea forticeps, Amyciaea forticeps, Angaeus pentagonalis, Borboropactus elephantus, Camaricus bipunctatus, Camaricus formosus , Camaricus khandalaensis, Camaricus siltorsus, Diaea bengalensis, Ebrechtella concinna, Ebrechtella tricuspidata, Henriksenia hilaris, Heriaeus horridus, Indoxysticus minutus, Loxobates kapuri, Mastira menoka, Misumena annapurna, Misumena greenae, Misumena indra, Misumena mridulai, Misumena ritujae, Misumenoides gwarighatensis, Misumenops khandalaensis, Monaeses mukundi, Monaeses pachpediensis, Monaeses parvati, Oxytate elongata, Oxytate virens, Ozyptila chandosiensis, Ozyptila maratha, Pasias marathas, Pistius kalimpus, Pistius robustus, Platythomisus sudeepi, Runcinia acuminata, Runcinia ghorpadei, Runcinia insecta, Runcinia khandari, Runcinia roonwali, Runcinia yogeshi, Strigoplus moluri, Strigoplus netravati, Synema decoratum, Tharrhalea evanida, Thomisus andamanensis, Thomisus ashishi, Thomisus beautifularis, Thomisus danieli, Thomisus elongatus, Thomisus granulifrons, Thomisus katrajghatus, Thomisus lobosus, Thomisus mimae, Thomisus okinawensis, Thomisus pateli, Thomisus pathaki, Thomisus pooneus, Thomisus projectus, Thomisus pugilis, Thomisus rajani, Thomisus shivajiensis, Thomisus sorajaii, Thomisus spectabilis, Thomisus viveki, Thomisus whitakeri, Tmarus jabalpurensis, Tmarus kotigeharus, Xysticus bharatae, Xysticus breviceps, Xysticus jabalpurensis, Xysticus joyantius, Xysticus kali, Xysticus khasiensis, Xysticus tikaderi

15. Manipur: Amyciaea forticeps, Camaricus formosus, Diaea bengalensis, Diaea dorsata, Massuria sreepanchamii, Misumena sp., Oxytate elongata, Oxytate virens, Runcinia acuminata, Runcinia insecta, Thomisus projectus, Tmarus sp.

16. Meghalaya: Amyciaea forticeps, Borboropactus elephantus, Camaricus formosus , Diaea subdola, Henriksenia hilaris, Indoxysticus minutus, Lysiteles mandali, Massuria sreepanchamii, Misumena mridulai, Misumena vatia, Ozyptila khasi, Runcinia insecta, Runcinia roonwali, Thomisus andamanensis, Thomisus shillongensis, Tmarus kotigeharus, Xysticus croceus, Xysticus joyantius, Xysticus kamakhyai, Xysticus khasiensis, Xysticus pynurus, Xysticus shillongensis, Xysticus shyamrupus

17. Mizoram: Camaricus formosus, Ebrechtella concinna, Oxytate virens, Ozyptila sp., Thomisus pugilis, Xysticus croceus

18. Odisha: Amyciaea forticeps, Bomis sp., Camaricus formosus, Camaricus khandalaensis, Indoxysticus minutus, Oxytate sp., Runcinia sp., Thomisus lobosus, Thomisus projectus, Thomisus pugilis, Thomisus sikkimensis

19. Punjab: Indoxysticus minutus, Misumena ganpatii, Monaeses parvati, Pistius tikaderi, Thomisus dyali, Thomisus ludhianaensis, Thomisus pugilis, Thomisus shivajiensis, Tmarus kotigeharus

20. Rajasthan: Bomis sp., Indoxysticus minutus, Mecaphesa celer, Ozyptila brevipes, Ozyptila chandosiensis, Ozyptila reenae, Synema sp., Thomisus 
andamanensis, Thomisus italongus, Thomisus lobosus, Thomisus projectus, Thomisus pugilis, Tmarus kotigeharus, Xysticus bengalensis

21. Sikkim: Epidius armatus, Pistius kalimpus, Runcinia bifrons, Thomisus rishus, Thomisus sikkimensis, Xysticus roonwali, Xysticus sikkimus

22. Tamil Nadu: Amyciaea forticeps, Angaeus pentagonalis, Bomis khajuriai, Camaricus formosus, Diaea pougneti, Dietopsa castaneifrons, Dietopsa parnassia, Epidius armatus, Epidius longimanus, Epidius mahavira, Henriksenia hilaris, Holopelus malati, Indoxysticus minutus, Lycopus trabeatus, Lysiteles catulus, Oxytate chlorion, Oxytate virens, Ozyptila theobaldi, Pagida salticiformis, Runcinia escheri, Runcinia insecta, Synema decoratum, Talaus opportunus, Thomisus beautifularis, Thomisus granulifrons, Thomisus laglaizei, Thomisus leucaspis, Thomisus lobosus, Thomisus pugilis, Thomisus rigoratus, Thomisus spectabilis, Tmarus fasciolatus, Tmarus soricinus, Xysticus sp.

23. Telangana: Thomisus projectus, Thomisus telanganaensis

24. Tripura: Amyciaea lineatipes, Camaricus formosus, Camaricus maugi

25. Uttar Pradesh: Camaricus formosus, Diaea subdola, Indoxysticus minutus, Lysiteles sp., Massuria roonwali, Mastira menoka, Misumena indra, Misumena mridulai, Misumena vatia, Ozyptila chandosiensis, Ozyptila manii, Pistius bhadurii, Runcinia insecta, Runcinia roonwali, Thomisus lobosus, Thomisus pooneus, Thomisus projectus, Thomisus pugilis, Thomisus sorajaii, Thomisus unidentatus, Xysticus $s p$.

26. Uttarakhand: Amyciaea forticeps, Bomis sp., Camaricus formosus, Diaea sp., Henriksenia hilaris, Heriaeus horridus, Indoxysticus minutus, Lysiteles brunettii, Lysiteles niger, Massuria roonwali, Mastira menoka, Misumena mridulai, Misumenoides naginae, Misumenops sp., Monaeses sp., Oxytate elongata, Ozyptila sp., Pistius barchensis, Pistius bhadurii, Pistius gangulyi, Pistius kanikae, Pistius robustus, Runcinia insecta, Synema decoratum, Thomisus dentiger, Thomisus lobosus, Thomisus onustus, Xysticus croceus, Xysticus jaharai, Xysticus joyantius, Xysticus kali, Xysticus shyamrupus

27. West Bengal: Amyciaea forticeps, Bomis bengalensis, Bomis calcuttaensis, Camaricus formosus, Camaricus khandalaensis, Camaricus siltorsus, Diaea bengalensis, Epidius armatus, Henriksenia hilaris, Indosmodicinus bengalensis, Indoxysticus minutus, Lysiteles brunettii, Massuria roonwali, Massuria sreepanchamii, Massuria uthoracica, Mastira nicobarensis, Misumena mickeyi, Oxytate greenae, Ozyptila khasi, Ozyptila manii, Ozyptila reenae, Pharta indica, Phrynarachne peeliana, Phrynarachne tuberosa, Pistius barchensis, Pistius gangulyi, Pistius kalimpus, Runcinia insecta, Stiphropus soureni, Strigoplus bilobus, Synema decoratum, Synema revolutum, Tharpyna indica, Thomisus andamanensis, Thomisus beautifularis, Thomisus bulani, Thomisus dhakuriensis, Thomisus elongatus, Thomisus katrajghatus,
Thomisus mimae, Thomisus nirmali, Thomisus projectus, Thomisus pugilis, Thomisus sikkimensis, Thomisus sorajaii, Thomisus spectabilis, Xysticus bengalensis, Xysticus bengdakus, Xysticus croceus, Xysticus himalayaensis, Xysticus hindusthanicus, Xysticus kali

\section{Checklist of Crab Spiders (Thomisidae) in Union Territories of India}

1. Andaman \& Nicobar Islands: Demogenes andamanensis, Angaeus pentagonalis, Borboropactus bituberculatus, Camaricus formosus, Mastira nicobarensis, Oxytate greenae, Phrynarachne ceylonica, Runcinia insecta, Runcinia kinbergi, Thomisus andamanensis, Thomisus armillatus, Thomisus onustus

2. Delhi: Camaricus formosus, Monaeses parvati, Oxytate elongata, Thomisus andamanensis, Thomisus unidentatus

3. Jammu \& Kashmir: Diaea subdola, Ebrechtella concinna, Ebrechtella sufflava, Ebrechtella tricuspidata, Lysiteles excultus, Misumena oblonga, Ozyptila trux, Psammitis setiger, Psammitis xysticiformis, Runcinia insecta, Thomisus elongatus, Thomisus lobosus, Thomisus nirmali, Thomisus projectus, Thomisus pugilis, Tmarus dejectus, Tmarus stellio, Xysticus audax, Xysticus cristatus, Xysticus maculiger, Xysticus mundulus

4. Ladakh: Misumena sp., Xysticus breviceps

\section{Lakshadweep: Thomisus pugilis}

6. Puducherry: Amyciaea forticeps, Henriksenia hilaris, Thomisus granulifrons, Thomisus spectabilis, Tmarus fasciolatus, Xysticus cristatus

\section{CONCLUSION}

The diversity of Thomisidae, commonly called crab spiders are listed in Indian states and union territories. More than two hundred species are recorded in almost all states and union territories in India except Nagaland and Dadra \& Nagar Haveli and Daman \& Diu. Out of 210 species recorded, 143 species are strictly endemic to India. Despite spiders being the most diverse group of predators and crucial to the health of terrestrial ecosystems, none of the species recorded in India is listed in IUCN Red List. An extensive survey for these spiders is urgently required.

\section{ACKNOWLEDGMENT}

We thank Dr. Theo Blick, Hummeltal, Denmark, and an editorial member of World Spider Catalog, for many suggestions regarding the distribution of crab spiders in India.

\section{REFERENCES}

Abhijith,APC.https://www.mysorenature.org/mysorenature/ Spiders-of-Mysore-Area, as on 15/10/2019.

Abhilash, R., \& Kumar, A.S. (2018). A survey of spider diversity on the bank of River Pampa at Poovathoor, 
Pathanamthitta District, Kerala. Trends Biosciences, 11(7), 1025-1028.

Adarsh, C.K., \& Nameer, P.O. (2016). A preliminary checklist of spiders (Araneae, Arachnida) in Chinnar Wildlife Sanctuary, Western Ghats, India. Journal of Threatened Taxa, 8(4), 8703-8713.

Agrawal, V.C., \& Ghose, R.K. (1995). Fauna of Conservation Areas, Fauna of Palamau Tiger Reserve, Zoological Survey of India, 8, 83-103.

Ahmed, A.F. (2018). A preliminary study on spider diversity in Matia, Goalpara District, Assam, India. International Journal of Zoology and Applied Biosciences, 3(4), 369-374.

Ambily, C.B., \& Antony, A. (2016). Diversity and distribution of spiders in agro ecosystem of Ernakulum, District, Kerala. The Journal of Zoology Studies, 3(5), 73-77.

Anjali \& Prakash, S. (2012). Diversity of spiders (Araneae) from semi arid habitat of Agra (India). Indian Journal of Arachnology, 1(2), 66-72.

Asima, A., Prasad, G. and Sudhikumar, A.V. (2020). Spider diversity of Kerala University Campus, Thiruvananthapuram, Kerala, India. Entomon, 45(3), 209-218.

Bastawade, D.B. (2002). Three new species from the spider families Amaurobiidae, Thomisidae and Salticidae (Araneae, Arachnida) from India. Journal of the Bombay Natural History Society, 99(2), 274-281.

Bastawade, D.B. (2008). Arachnida, Scorpionida, Araneae and Opiliones. In, Fauna of Lonar Wildlife Sanctuary. Conservation area Series, Zoological Survey of India, Kolkata, 37, 133-153.

Bastawade, D.B., \& Borkar, M. (2008). Arachnida (orders Scorpiones, Uropygi, Amblypygi, Araneae and Phalangida). In, Fauna of Goa, State Fauna Series, Zoological Survey of India, Kolkata, 16, 211-242.

Bastawade, D.B., \& Khandal, D. (2006). Arachnida, Araneae (spiders). In, Fauna of Sanjay Gandhi National Park (Invertebrates) Borivali, Mumbai (Maharashtra), Conservation Area Series, Zoological Survey of India, Kolkata, 26, 139-184.

Bastawade, D.B., \& Sharma, R.M. (2002). New record of spider Strigoplus netravati from Maharashtra with additional morphological descriptions (Araneae, Thomisidae). Bionotes, 4(4), 91.

Basu, B.D. (1963). A new spider of the family Thomisidae (Araneae) from India. Science and Culture, 29, 606607.

Basu, B.D. (1964a). Diagnosis of two new species of Pistius (Thomisidae, Araneae, Arachnida) from India. Journal of the Bengal Natural History Society, 32, 104-109.
Basu, B.D. (1964b). Morphology of an Indian spider of the family Thomisidae (Araneae, Arachnida). Science and Culture, 30, 154-155.

Basu, B.D. (1965a). Four new species of the spider genus Pistius Simon (Arachnida, Araneae, Thomisidae) from India. Proceedings of the Zoological Society, Calcutta, 18, 71-77.

Basu, B.D. (1965b). On the description of two new spiders of the family Thomisidae (Arachnida, Araneae) from India. Journal of the Asiatic Society of Bengal, 5, 2326.

Basu, K.C. (1979). On a new spider of the genus Xysticus Koch, 1835 (Thomisidae, Arachnida) from Nainital, India. Journal of the Zoological Society of India, 28, 149-150.

Basumatary, P., \& Brahma, D. (2017). Checklist of spiders from Chakrashila Wildlife Sanctuary, Assam. International Journal of Zoology Studies, 2(5), 22-26.

Benjamin, S.P. (2011). Phylogenetics and comparative morphology of crab spiders (Araneae, Dionycha, Thomisidae). Zootaxa, 3080(1), 1-108.

Benjamin, S.P. (2017). Distributional and taxonomic notes on the crab spider genus Epidius with descriptions of five new species (Araneae, Thomisidae). Journal of Natural History, 51(9-10), 469-485.

Benjamin, S.P., Dimitrov, D., Hormiga, G., \& Gillespie, R.G. (2008). Family ties, molecular phylogeny of crab spiders (Araneae, Thomisidae). Cladistics, 24, 708722.

Bhandari, R., \& Gajbe, P. (2001). Description of three new species of spiders of the genera Thomisus Walckenaer, Oxyptila Simon and Xysticus Koch (Araneae, Thomisidae) from Madhya Pradesh, India. Records of the Zoological Survey of India, 99, 81-85.

Bhat, P.S., Srikumar, K.K., Raviprasad, T.N. (2013). Seasonal diversity and status of spiders (Arachnida, Araneae) in cashew ecosystem. World Applied Sciences Journal, 22(6), 763-770.

Bhattacharya, A., Chetri, M., \& Sarkar, P. (2017). Spider diversity in different habitats at Jaintia Hills of Meghalaya. International Journal of Life Sciences, 5(4), 613-619.

Bhattacharya, G.C. (1934). Notes on observation of some peculiar habits of an antmimicking spider Amyciaea forticeps (Cambridge). Journal of the Bombay Natural History Society, 37(1), 233.

Biswas, A.T. (1977). A new species of spider of the genus Platythomisus Simon (family, Thomisidae) from Coorg, Karnataka, India. Journal of the Bombay Natural History Society, 74, 332-334.

Biswas, B., \& Biswas, K. (1992). Araneae, Spiders. In, State Fauna Series. Fauna of West Bengal, Zoological Survey of India, Kolkata, 3, 357-500. 
Biswas, B.K., \& Biswas, K. (2004). Araneae, Spiders. In, Fauna of Manipur, State Fauna Series. Zoological Survey of India, Kolkata, 10(2), 25-46.

Biswas, B., \& Biswas, K. (2006). Araneae, Spiders. In, Fauna of Arunachal Pradesh, State Fauna Series, Zoological Survey of India, Kolkata, 13(2), 491-518.

Biswas, B., \& Biswas, K. (2007). Araneae, Spiders. In, Fauna of Mizoram, State Fauna Series. Zoological Survey of India, Kolkata, 14, 455-475.

Biswas, B., \& Biswas, K. (2010). Araneae, Spiders. In, Fauna of Uttarakhand, State Fauna Series, Zoological Survey of India, Kolkata, 18(3), 243-282.

Biswas, B., \& Majumder, S.C. (1995). Araneae, Spider. In, Fauna of Meghalaya, State Fauna Serie, Zoological Survey of India, Kolkata, 4(2), 93-128.

Biswas, B., \& Mazumder, S.C. (1981). Description of two new species of crab-spiders of the genera Diaea and Bomis (Family, Thomisidae) from India. Bulletin of Zoological Survey of India, 4, 271-275.

Biswas, B., \& Roy, R. (2005a). Description of three new species of the genera Thomisus Walckenaer, Cheiracanthium Koch, C.L., \& Tinus Cambridge of the families Thomisidae, Clubionidae and Pisauridae from India. Records of the Zoological Survey of India, 105(3-4), 37-42.

Biswas, B., \& Roy, R. (2005b). Description of a new species of spider genus Camaricus (Thomisidae, Araneae) from India. Records of the Zoological Survey of India, 105(1-2), 165-167.

Biswas, B., \& Roy, R. (2008). Description of six new species of spiders of the genera Lathys (Family, Dictynidae), Marpissa (Family, Salticidae), Misumenoides (Family, Thomisidae), Agroeca (Family, Clubionidae), Gnaphosa (Family, Gnaphosidae) and Flanona (Family, Lycosidae) from India. Records of the Zoological Survey of India, 108, 43-57.

Bonnet, P. (1959). Bibliographia araneorum. Analyse méthodique de toute la littérature aranéologique jusqu'en 1939. Tome II. Systématique des araignées (Étude par ordre alphabétique) (5me partie, T-Z). Douladoure, Toulouse, pp. 4231-5058.

Caleb, J.T.D. (2020a). Spider (Arachnida, Araneae) fauna of the scrub jungle in the Madras Christian College campus, Chennai, India. Journal of Threatened Taxa, 12(7), 15711-15766.

Caleb, J.T.D. (2020b). Spiders (Arachnida, Araneae) from the vicinity of Araabath Lake, Chennai, India. Journal of Threatened Taxa, 12(1), 15186-15193.

Caleb, J.T.D., \& Karthikeyani, R. (2020). JoTT Checklist of the spiders of Tamil Nadu (v1.0), 10 February 2020.
Caleb, J.T.D., \& Sankaran, P.M. (2021). Araneae of India, version 2021. https://indianspiders.in/ retrieved on 20 May, 2021.

Caporiacco, L.di (1935). Aracnidi dell'Himalaia e del Karakoram, raccolti dalla Missione italiana al Karakoram (1929-VII). Mem. Soc. Ent. Italiana, Genova, 13, 161-263.

Cardoso, P., Crespo, L.C., Silva, I., Borges, P., \& Boieiro, M. (2018). Xysticus grohi. The IUCN Red List of Threatened Species 2018: e.T58053533A58061357.

Chandra, K., Bharti, D., Kumar, S., Raghunathan, C., Gupta, D., Alfred, J.R.B. and Chowdhury, B.R. (2021). Faunal Diversity in Ramsar Wetlands of India. Zoological Survey of India, Kolkata, pp. 1-292.

Chapke, S.P. (2012). Spider diversity of agroecosystem in Washim district (MS) India. Indian Journal of Research, 1(7), 73-76.

Chauhan, R., Sihag, V., \& Singh, N.P. (2009). Distribution and biocontrol potential of chosen spiders. Journal of Biopesticides, 2(2), 151-155.

Chetia, P., \& Kalita, D.K. (2012). Diversity and distribution of spiders from Gibbon Wildlife Sanctuary, Assam, India. Indian Journal of Arachnology, 1(1), 130-142.

Chetry, A., \& Moran, J. (2019). Diversity of Namsai District, Arunachal Pradesh, India. International Journal of Basic and Applied Research, 9(7), 343-351.

Choudhury, S.R., Siliwal, M., \& Das, S.K. (2019). Spiders of Odisha, a preliminary checklist. Journal of Threatened Taxa, 11(9), 14144-14157.

Chowdhury, S., Boopathi Bhattacharjee, T.B., Dey, J.K., \& Bhattacharjee, J. (2017). Diversity of predatory spider and their species composition in rice ecosystem in Kolasib district of Mizoram. Innovative Farming, 2(1), 12-18.

Das, S., Kalita, J., \& Mahanta, N. (2019). New report of Phrynarachne ceylonica (O. Pickard-Cambridge, 1884) (Araneae, Thomisidae) from Assam, India. International Journal of Scientific Research and Reviews, 8(2), 985-990.

Das, S., Bhattacharjee, R., \& Saikia, P.K. (2015). Comprehensive checklist of diurnal spider diversity in Guwahati metropolitan area, Kamrup, Assam. Tropical Zoology, 5, 121-131.

Dash, M., \& Sivaperuman, C. (2021a). New distributional report of Borboropactus bituberculatus Simon 1884 from Nicobar Islands, India. World News of Natural Sciences, 36, 1-8.

Dash, M., \& Sivaperuman, C. (2021b). Notes on the presence of Phrynarachne ceylonica (O. PickardCambridge, 1884) from the Andaman and Nicobar Islands. World News of Natural Sciences, 35, 48-55. 
De, K., \& Palita, S.K. (2018). A checklist of spiders from six sacred groves in Southern Odisha, India. Serket, 16(1), 30-40.

Deshmukh, U.S., \& Tekade, A.P. (2019). A report on the diversity of spider fauna from Charghad river basin of Morshi, Amravati India. Bioscience Biotechnology Research Communications, 12(3), 809-813.

Deshpande, A., \& Paul, R. (2016). Preliminary study on spiders of Gulbarga, Karnataka State. International Journal of Environment, Agriculture and Biotechnology, 1(4), 680-686.

Dey, A., Debnath, S., Debbarma, B., Chaudhuri, P.S. (2013). A preliminary study on spider diversity from a house hold garden (artificial mixed plantation) in West Tripura, India. Journal of Research in Biology, 3:10091017

Dhali, D.C., Sunil Jose, K., \& Sureshan, P.M. (2019). Arachnida , Araneae (Spiders). In, Fauna of Malabar Wildlife Sanctuary, Kozhikode, Kerala, Consevation Area Series, Zoological Survey of India, Kolkata, 62, 155-189.

Diksha, Khan, R.A., Sultana, A., \& Das, S.K. (2018). A new spider record of genus Thomisus Walckenaer, 1805 (Araneae, Thomisidae) from India. Serket, 16(2), 96-99.

Ekka, A., \& Kujur, R. (2015). Spider diversity of Ram Jharna, Raigarh district, Chhattisgarh, India. Research Journal of Pharmacy \& Technology, 8(7), 813-819.

Gajbe, P. (2003a). A checklist of spiders (Arachnida, Araneae) of Jabalpur, Madhya Pradesh, India. Records of the Zoological Survey of India, 101(3-4), 43-47.

Gajbe, P. (2003b). Checklist of spiders (Arachnida, Araneae) of Madhya Pradesh and Chhattisgarh. Zoos' Print Journal, 18(10), 1223-1226.

Gajbe, P. (2004a). Description of three new species of crab spiders (Araneae, Thomisidae) from Madhya Pradesh, India. Records of the Zoological Survey of India, 103(1-2), 123-130.

Gajbe, P. (2004b). Fauna of protected areas-11. Spiders of Pench Tiger Reserve, Madhya Pradesh. Zoos' Print Journal, 19(9), 16-24.

Gajbe, P. (2004c). A preliminary list of spider fauna of Pachmarhi Biosphere Reserve, Madhya Pradesh. Zoos' Print Journal, 19(3), 1414-1415.

Gajbe, P.U. (2004d). Spiders of Jabalpur, Madhya Pradesh (Arachnida, Araneae). Records of the Zoological Survey of India, Kolkata, Occasional Paper, 227, 1154.

Gajbe, U.A. (1995a). Arachnida, Spiders. In, Fauna of Conservation Areas No.7, Fauna of Kanha Tiger Reserve, Madhya Pradesh. Zoological Survey of India, Kolkata, pp. 27-30.
Gajbe, U.A. (1995b). Arachnida, Spiders. In, Fauna of conservation area No.6. Fauna of Indravati Tiger Reserve (Arachnida, spiders). Zoological Survey of India, Kolkata, pp. 53-56.

Gajbe, U.A. (2005). Studies on some spiders of the family Thomisidae (Araneae, Arachnida) from Madhya Pradesh, India. Records of the Zoological Survey of India, 105(3-4), 57-80.

Gajbe, U.A. (2007). Araneae, Arachnida. In, Fauna of Madhya Pradesh (including Chhattisgarh), State Fauna Series. Zoological Survey of India, Kolkata, 15(1), 419-540.

Gajbe, U.A. (2008a). A new species of Misumena spider (Araneae, Thomisidae) from Jabalpur, Madhya Pradesh, India. Records of the Zoological Survey of India, 108(1), 63-65.

Gajbe, U.A. (2008b). A new species of Dieta spider (Araneae, Philodromidae) from Jabalpur, Madhya Pradesh, India. Records of the Zoological Survey of India, 108(1), 59-61.

Gajbe, U.A., \& Gajbe, P. (1999a). A new species of spider of the genus Tmarus Simon (Araneae, Thomisidae) from Madhya Pradesh, India. Records of the Zoological Survey of India, 97(3), 141-143.

Gajbe, U.A., \& Gajbe, P. (1999b). On two new species of spiders of the genus Xysticus Koch (Araneae, Thomisidae) from Madhya Pradesh, India. Records of the Zoological Survey of India, 97(3), 145-148.

Gajbe, U.A., \& Gajbe, P. (2000a). A new species of the genus Runcinia Simon (Araneae, Thomisidae) from Madhya Pradesh, India. Records of the Zoological Survey of India, 98, 155-157.

Gajbe, U.A., \& Gajbe, P. (2000b). A new species of spider of the genus Thomisus Walckenaer (Araneae, Thomisidae) from Madhya Pradesh, India. Records of the Zoological Survey of India, 98, 55-57.

Gajbe, U.A., \& Rane, P.D. (1992). A new Monaeses spider from Madhya Pradesh, India (Araneae, Thomisidae). Records of the Zoological Survey of India, 91, 395397.

Gajbe, U.A., \& Sharma, H.S. (1994). On some spiders (Araneae, Arachnida) from Bastar district (Madhya Pradesh) India. Records of the Zoological Survey of India, 94 (2-4), 233-245

Ganesh Kumar, M., \& Velusamy, R. (1996). Composition of spider in rice ecosystem of Tamil Nadu. Madras Agricultural Journal, 83(7), 448-451.

Gerlach, J. (2014). Firmicus insularis. The IUCN Red List of Threatened Species 2014: e.T196510A2458889.

Ghosh, N., Biswas, R., \& Mitra, A. (2018). Species diversity, abundance and habitat association of spiders with relation to their guild composition in different habitats of North Bengal Wild Animals Park (Bengal 
Safari). International Journal of Life Sciences, 6(4), 911-918.

Gupta, N., \& Siliwal, M. (2012). A checklist of spiders (Arachnida, Araneae) of Wildlife Institute of India campus, Dehradun, Uttarakhand, India. Indian Journal of Arachnology, 1(2), 73-91.

Gupta, R., Devi, O.S., \& Islam, M. (Eds.) (2015). Common spiders from select protected areas of upper Assam. Assam State Biodiversity Board Rehabari, Guwahati, pp.186.

Halarnkar, M.M., \& Pai, I.K. (2018). Distribution, diversity and ecology of spider species at two different habitats. International Journal of Environmental Sciences \& Natural Resources, 8(5), 162-167.

Hore, U., \& Uniyal, V.P. (2008a). Effect of prescribed fire on spider assemblage in Terai grasslands, India. Turkish Journal of Arachnology, 1(1), 15-36.

Hore, U., \& Uniyal, V.P. (2008b). Diversity and composition of spider assemblages in five vegetation types of the Terai Conservation Area, India. Journal of Arachnology, 36(2), 251-258.

ICAR-NBAIR (2019). Annual report 2018-2019. ICARNational Bureau of Insect Resources, Bengaluru, India, pp. 122.

Jose, A.C., Sudhin, P.P., Prasad, P.M., Sreejith, K.A. (2018). Spider diversity in Kavvayi river basin, Kerala, Southern India. Current World Environment, 13(1), 100-112.

Jose, J., Ramachandran, K.K., \& Nair, P.V. (2007). A preliminary overview and checklist of the spider fauna of Myristica swamp forests of southern Kerala, India. Newsletter of the British Arachnological Society, 109, 12-14.

Joseph, A.R and Premila, K.S. (2016). A study on the richness of spider fauna in rice ecosystem. Journal of Entomology and Zoology Studies, 4(2), 425-430.

Joseph, M.M., Paul, J., Sankaran, P.M., \& Sebastian, P.A. (2017). Preliminary results on the spider fauna (Arachnida, Araneae) of the high altitude Shola ecosystem in the Western Ghats. Proceedings of the National Conference on Ecology Sustainable Development and Wildlife Conservation, pp. 41-49.

Kadam, G., \& Rajkumar, M. (2020). Exploring the "tigers of microhabitat" in SACON campus. SACON News, 17(4), 6-10.

Kananbala, A., Bhubaneshwari, M., \& Siliwal, M. (2018). A checklist of spiders (Arachnidae, Araneae) of Manipur, India with some first records and a new species Conothele khunthokhanbi (Family, Ctenizidae). Journal of Entomology and Zoology Studies, 6(5), 2209-2214

Kapoor, V. (2008). Effects of rainforest fragmentation and shade-coffee plantations on spider communities in the
Western Ghats, India. Journal of Insect Conservation, 12, 53-68.

Karthikeyani, R. (2013). Biodiversity of spiders (Araneae) in Kumbakarai Falls, Periyakulam Taluk, Theni District, Tamil Nadu, South India. Ph. D. thesis, Madurai Kamaraj University, Madurai, pp. 288.

Karthikeyani, R., Caleb, J.T.D., Gajbe, U.A., \& Muthuchelian, K. (2017). Checklist of spiders (Arachnida, Araneae) of the State of Tamil Nadu, India. Munis Entomology \& Zoology, 12(1), 180-193.

Kashmeera, N.A., Drisya-Mohan, O.M., \& Sudhikumar, A.V. (2020). Spiders of rocky desert in Kailana, Rajasthan, India. Serket, 17(3), 201-206.

Kaur, M., Das, S.K., Anoop, K.R., \& Siliwal, M. (2014). Preliminary checklist of spiders of Keoladeo National Park, Bharatpur, Rajasthan with first record of Ptocasius $\quad$ strupifer Simon, $1901 \quad$ (Araneae, Salticidae) from India. Munis Entomology \& Zoology, 9(1), 501-509.

Keswani, S. (2014). Diversity, population and microhabitat used by spiders in citrus agroecosystem. Indian Journal of Arachnology, 3(2), 90-101.

Keswani, S., Hadole, P., \& Rajoria, A. (2012). Checklist of spiders (Arachnida, Araneae) from India. Indian Journal of Arachnology, 1(1), 1-129.

Khan, A.A. (2006). Relative abundance of spider fauna of rice ecosystem. SAARC Journal of Agriculture, 4, 159166.

Khan, A.A. (2009). Biodiversity of spider fauna (Arachnida, Araneae) in horticultural ecosystem of Kashmir. Indian Journal of Ecology, 36(1), 59-64.

Khan, A.A., \& Misra, D.S. (2003). Studies on qualitative and quantitative composition of spider fauna in rice ecosystem of eastern Uttar Pradesh. Plant Protection Bulletin, 55(1-2), 35-41.

Khan, A.A., \& Rather, A.Q. (2012). Diversity and foraging behaviour of spiders (Arachnida, Araneae) in the temperate maize ecosystem of Kashmir. Journal of Biological Control, 26(2), 179-189.

Kokilamani A.L., Lokeshkumar, P., Rakesh, B.O., Sahana, R., Geetha, H.C. (2019). A preliminary study on diversity of spiders from Tumkur University Campus, Tumakuru, India. International Journal of Advanced Scientific Research and Management, 4(2), 84-87.

Kujur, R., \& Ekka, A. (2012). Inventorization of Spider fauna of IndraVihar Park, Raigarh, Chhattisgarh, India. IOSR Journal of Environmental Science, Toxicology and Food Technology, 1(2), 20-26.

Kujur, R., \& Ekka, A. (2016). Exploring the spider fauna of Gomarda Wildlife Sanctuary, Chhattisgarh, India. International Research Journal of Biological Sciences, 5(6), 31-36. 
Kumar, A., Kanaujia, A., Kumar, A., Kumar, V., \& Mishra, H. (2017a). Diversity of spiders in Kukrail Reserve Forest, Lucknow, Uttar Pradesh, India. Journal of Environmental Science and Technology, 4(5), 42-45.

Kumar, A., Kanaujia, A., Kumar, A., Kumar, V., \& Mishra, H. (2017b). Araneofauna of Nawabganj bird sanctuary, Unnao, Uttar Pradesh, India. Journal of Entomology and Zoology Studies, 5(4), 1952-1955

Kumar, D., \& Shivakumar, M.S. (2006). Seasonal abundance of spiders in pigeonpea agroecosystem. Indian Journal of Environmental Science, 10(1), 4346.

Kumar, D., \& Yashkamal, K. (2011). Study of prey spectrum of social spider Stegodyphus sarasinorum (Karsch) (Araeneae, Eresidae) and its potential as biological control agent. Proceeding of the National Academy of Science, India, Section B, 81(2), 171-179.

Kumari, K. (1983). Taxonomy of spiders (Arachnida, Aranceae) from northern India. M. Phil. Thesis, Punjabi University, Patiala, Punjab.

Kumari, M., \& Mittal, O.P. (1994). A new species of crab spiders of the genus Misumena (Family, Thomisidae) from India. Bionature, 14, 29-31.

Kumari, M., \& Mittal, O.P. (1997). Two new species of crab spiders of the genus Thomisus (family, Thomisidae) from India. Bionature 17, 31-33.

Kumari, M., \& Mittal, O.P. (1999). A new species of crab spiders of the genus Pistius (Araneae, Thomisidae) from India. Research Bulletin of the Panjab University, 48, 17-19.

Kumari, V., Saini, K.C., \& Singh, N.P. (2017). Diversity and distribution of spider fauna in arid and semi-arid region of Rajasthan. Journal of Biopesticides, 10(1):17-24.

Lanka, L.P., Kamble, S.S., \& Bodkhe, A.K. (2017). An Addition to spider fauna from the vicinity of Radhanagari Wildlife Sanctuary of Kolhapur District. International Journal of Scientific Engineering and Research, 5(7), 280-283.

Lawania, K.K., \& Mathur, P. (2014a). Baseline studies on the spider fauna (Araneae) of Braj region (BrajBhoomi), India. International Journal of Basic and Applied Biology, 2(1), 137-141.

Lawania, K.K., \& Mathur, P. (2014b). Diversity and distribution of spider fauna in and around the Taj Mahal and Taj Protected Forest, Agra (U.P.), India. International Journal of Basic and Applied Biology, 2(2), 111-114.

Lawania, K.K., \& Mathur, P. (2014c). On the diversity of spiders in and around Sur-Sarovar Bird Sanctuary, Agra (U.P.), India. International Journal of Basic and Applied Biology, 2(3), 189-194.
Lawania, K.K., \& Mathur, P. (2014d). Diversity and distribution of spiders in and around Vrindavan, Mathura, (UP), India. International Journal of Basic and Applied Biology, 2(2), 115-119.

Lawania, K.K., \& Mathur, P. (2017). Biodiversity and habit preference of spider fauna in eastern region of Rajasthan and its catchment area. International Journal of Scienticifc Development and Research, 2(6), 475-484.

Lawania, K.K., \& Trigunayat, M.M. (2015). A comparative study of the spider (Araneae) fauna in Keoladeo National Park (KNP), Nahargarh Wildlife Sanctuary (NWS) and Sur-sarovar Bird Sanctuary (SBS), India. International Journal on Agricultural Sciences, 6(1), 141-146.

Leardi in Airaghi, Z. (1901a). Aracnidi d'Almora. Atti della Societa Italiana di Scienze Naturali e del Museo Civico di Storia Naturale di Milano, 40, 85-94.

Leardi in Airaghi, Z. (1901b). Aracnidi di Mahé e Kandy. Atti della Societa Italiana di Scienze Naturali e del Museo Civico di Storia Naturale di Milano, 40, 345373.

Lehtinen, P.T. (2004). Taxonomic notes on the Misumenini (Araneae, Thomisidae, Thomisinae), primarily from the Palaearctic and Oriental regions. In, Logunov, D.V., \& Penney, D. (eds.) European Arachnology 2003 (Proceedings of the 21st European Colloquium of Arachnology, St.-Petersburg, 4-9 August 2003). Arthropoda Selecta, Special Issue, 1, 147-184.

Maheshwari, N.R., Patil, S.R., \& Choda, M.Z. (2018). Spider fauna from north Maharashtra, India. Flora \& Fauna, 24(1), 163-173.

Majumder, S.C. (2004). Taxonomic studies of some spiders from mangrove and semi-mangrove areas of Sundarban. Memoirs of the Zoological Survey of India, 20(2), 1-42.

Majumder, S.C. (2005). Studies on some spiders from eastern coastal region of India. Memoirs of the Zoological Survey of India, 20(3), 1-57.

Malamel, J.J., \& Sudhikumar, A.V. (2017). First record of Epidius parvati Benjamin, 2000 (Araneae, Thomisidae) from Pathiramanal Island, India. Check List, 13(3, 2114), 1-4.

Malamel, J.J. \& Sudhikumar, A.V. (2020). An investigation of diversity and bioecology of araneofauna of Pathiramanal Island in Vembanad Lake, a Ramsar site, Kerala, India. Zoology and Ecology, 30(2), 126-137.

Malhotra, G.S., Neera, K., \& Saxena, M.M. (2019). Spider diversity and abundance in different habitats of UpperNorthern Rajasthan. Essence International Journal for Environmental Rehabilitation and Conservation, 10 (1), 1-14.

Malik, S., Das, S.K., \& Siliwal, M. (2015). Spider (Arachnida, Araneae) fauna of Delhi with first report 
of cobweb spider, Argyrodes bonadea (Karsch, 1881) from India. Indian Journal of Arachnology, 4(2), 3136.

Malik, V., \& Goyal, V. (2017). Biodiversity of spiders in different habitats of Western Haryana, India. Journal of Entomology and Zoology Studies, 5(4), 822-825.

Mathew, E.V., Sudhikumar, A., \& Sebastian, P.A. (2014). Vertical stratification of spiders in Kuttanad rice agroecosystem, Kerala. Journal of Biological Control, 28(2), 62-67.

Meshram, A. (2011). Spiders (Arachnida, Araneae) from Toranmal Sanctuary, Maharashtra, India. EInternational Scientific Research Journal, 4, 326-334.

Mohapatra, A.K., Biswas, T., \& Parida, S.P. (2014). Spider Diversity in RIE campus. RIE, Bhubaneswar, pp. 76.

More, S. (2015a). Diversity of spider fauna from Bamnoli region of Koyna Wildlife Sanctuary. International Journal of Science and Research, 4(6), 1690-1693.

More, S.B. (2015b). Spider diversity from Vakoba, Devrai region of Radhanagari Wildlife Sanctuary. International Journal of Science and Research, 4(6), 179-181.

More, S., \& Sawant, V. (2013). Spider fauna of Radhanagari Wildlife Sanctuary, Chandoli National Parkandkoyna Wildlife Sanctuary. Indian Journal of Arachnology, 2(1), 81-92.

Mubeen, M., \& Basavarajappa S. (2018). Density, abundance and per cent occurrence of spider species (Arachnida, Araneae) in and around Mysore city, Karnataka, India - a case study. IOSR Journal of Pharmacy and Biological Sciences, 13(3), 31-40.

Murali, S., Jalali, S.K., Shylesha, A.N., Shivalinga Swamy, T.M., \& Gandhi Gracy, R. (2017). Predatory spider fauna in brinjal crop their abundance and composition. Journal of Entomology and Zoology Studies, 5(5), 675681

Nalini Bai, G., \& Ravindranatha, B.P. (2012). Spider diversity in IISC, Bangalore, India. Indian Journal of Arachnology, 1(2), 50-58.

Nautiyal, S., Khan, Y.D.I., Kaechele, H., \& Bhaskar, K. (2017). Diversity and distribution of spiders in Gogi, Yadgir District, a semi-arid landscape in southern India. International Journal of Ecology and Environmental Sciences, 43(3), 195-204.

Nerlekar, A.N., Warudkar, A.M., Gowande, G.G., Salve, S.S., Raut, A., Patankar, S.R., \& Nalavade, S.B. (2016). A review of the faunal diversity of the Fergusson College campus, Pune, India. Zoo's Print, 31(10), 4-25.

Nijagal, B.S., Padma, S., Michael, A., \& D'souza, L. (2020). Species composition, density, abundance and percent occurrence of spiders at three different sites of
Mysore city, Karnataka. IOSR Journal of Pharmacy and Biological Sciences, 15(3), 23-29.

Nyffeler, M., \& Birkhofer, K. (2017). An estimated 400800 million tons of prey are annually killed by the global spider community. The Science of Nature, 104, 30, 1-12.

Palem, H., Kanike, S., Purushottam, V.R.S. (2016). Diversity of spider fauna (Arachnida, Araneae) in different ecosystems, Eastern Ghats, Southern Andhra Pradesh, India. South Asian Journal of Life Science, 4(2), 51-60.

Panda, S., Mishra, S., Priyadarshini, D., \& Parida, P. (2011). Spiders of Nandankanan. Forest Department, Government of Odisha, pp. 64.

Pande, S., Mahabal, A., Datar, M., Sharma, R.M., Pati, S.K., Deshpande, P., Bastwade, D.B., Padhye, A., Kulkarni, A., Joshi, R., Pandit, R., Majumdar, N., Mhaske, P., Chhaya, K., Patil, R., \& Patwardhan, A. (2013). Bio-diversity of the Parvati-Panchgaon hills, A habitat island in Pune metropolis. Ela Journal, 2(1), 23-41.

Pandit, R., \& Dharwadkar, M. (2020). Preliminary checklist of spider fauna (Araneae, Arachnida) of Chandranath Hill, Goa, India. Journal of Threatened Taxa, 12(11), 16597-16606.

Pandit, R., \& Pai, I. (2017). Spiders of Taleigao Plateau, Goa, India. Journal of Environmental Science and Public Health, 1(4), 240-252.

Parasharya, B.M., \& Pathan, V.A. (2013). Diversity of spider fauna in lucerne (Medicago sativa L.). Journal of Biological Control, 27(4), 253-259.

Parmar, B.M. (2013). The spider diversity from different habitats around Biosciences, Vallabh Vidyanagar. International Journal of Science and Research, 4(10), 1985-1988.

Parmar, B.M. (2018). Preliminary study of spiders (Order, Araneae) from Satlasana taluka. International Journal of Pharmacy and Biological Sciences, 8(3), 735-740.

Parmar, B.M., \& Patel, K.B. (2017). Preliminary study of spiders (Order, Araneae) from Taranga Hills. International Journal of Science and Research, 6(11), 23-25.

Parmar, B.M., Acharya, A.V.R.L.N. (2015). The spider fauna of Pariej wetland, Gujarat, India. International Journal of Science and Research, 4(10), 1028-1033.

Parmar, B.M., Patel, K.B., Joshi, J.D., \& Chaudhari, N.R. (2015). Faunastic study of spider diversity from islands and costal areas of Gulf of Kutch, India. Life Science Leaflets, 67, 12-23.

Patel, B.H. (2003a). Fauna of Protected Areas - A preliminary list of spiders with the descriptions of three new species from Parambikulum Wildlife sanctuary, Kerala. Zoos’ Print Journal, 18(10), 1207-1212. 
Patel, B.H. (2003b). Spiders of Vansada National Park, Gujarat. Zoos’ Print Journal, 18(4), 1079-1083.

Patel, B.H., \& Pillai, G.K. (1988). Studies on the spider fauna of groundnut fields in Gujarat, India. Journal of Biological Control, 2(2), 83-88.

Patil, S.R. (2012). Spiders of Jabalpur district (Arachnida, Araneae), updated checklist 2011. Indian Journal of Arachnology, 1(1), 143-149.

Patil, S.R., Sambath, S.and Bhandari, R. (2013). Preliminary study on spider fauna (Arachnida, Araneae) of Singhori Wildlife Sanctuary, District Raisen, Madhya Pradesh, India. Bugs Revised All, No 20, 8-11.

Picard-Cambridge, O. (1885). Araneidea. In, Scientific results of the second Yarkand mission; based upon the collections and notes of the late Ferdinand Stoliczka, Ph.D. Government of India, Calcutta, pp. 115.

Pocock, R.I. (1901). Descriptions of some new species of spiders from British India. Journal of the Bombay Natural History Society, 13, 478-498.

Pocock, R.I. (1904). Arachnida. In, Fauna and geography of the Maldive and Laccadive Archipelagoes. London, 2, 797-805.

Pooja, A., Anilkumar, Quasin, S., Lekshmi, S., \& Uniyal, V.P. (2019). Spider fauna of Navdanya Biodiversity Farm, Uttarakhand, India. Indian Forester, 145(4), 392-397.

Prajapati, D.A., Patel, K.R., Munjpara, S.B., Chettiar, S.S., \& Jhala, D.D. (2016). Spiders (Arachnida, Araneae) of Gujarat University Campus, Ahmedabad, India with additional description of Eilica tikaderi (Platnick, 1976). Journal of Threatened Taxa, 8(11), 9327-9333.

Prashanthakumara, S.M \& Venkateshwarlu, M. (2017). Preliminary study of spiders (Araneae, Arachnida) in Gudavi Bird Sanctuary, Shivamogga, Karnataka. International Journal of Recent Scientific Research, 8(1), 19277-19281.

Pravalikha, G.B., \& Srinivasulu, C. (2015). A new species of genus Thomisus Walckenaer, 1805 (Araneae, Thomisidae) from Telangana, India and a detailed description of Thomisus projectus Tikader, 1960. Journal of Threatened Taxa, 7(3), 7000-7006.

Priyadarshini, N., Kumari, R., Pathak, R.N., \& Pandey, A.K. (2015). Biodiversity and community structure of spiders in Saran, part of Indo-Gangetic Plain, India. Asian Journal of Conservation Biology, 4(2), 121-129.

Priyadarshini, N., Kumari, R., Kumar, A. and Pandey, A.K. (2018). Diversity and seasonal variation of spiders community in a tropical region, a case study in Saran, Bihar, India. Ecology, Environment and Conservation Paper, 24(1), 263-269.

Punjoo, S., \& Bhat, G.A. (2015). First report of spiders (Arachnida, Araneae) from Dachigam National Park,
Kashmir, India. Asian Journal of Conservation Biology, 2(2), 707-719.

Quasin, S., \& Uniyal, V.P. (2010). Preliminary investigation of spider diversity in Kedarnath Wildlife sanctuary, Uttarakhand, India. Indian Forester, 136(10), 1340-1345.

Rajeevan, S., Kunnath, S.M., Varghese, T., \& Kandambeth, P.P. (2019). Spider diversity (Arachnida, Araneae) in different ecosystems of the Western Ghats, Wayanad Region, India. South Asian Journal of Life Science, 7(2), 29-39.

Rao, K.T., Bastawade, D.B., Javed, S.M.M., \& Krishna, I.S.R. (2006). Description of two new species of spiders of the genus Poecilotheria Simon (Araneae, Theraphosidae) and Tmarus Simon (Araneae, Thomisidae) from Nallamalai Hills, eastern Ghats, Andhra Pradesh, India. Records of the Zoological Survey of India, 106(1), 49-54.

Raychaudhuri, D., Saha, S., \& Roy, T.K. (2016). Spiders, a proficient candidate in practising IPM for Darjeeling Tea. World Science News, 38, 1-62.

Reddy, T.S., \& Patel, B.H. (1992). Two new species of the genus Thomisus Walckenaer (Araneae, Thomisidae) from coastal Andhra Pradesh. Journal of Bombay Natural History Society, 88, 268-272.

Reimoser, E. (1934). Araneae aus Süd-Indien. Revue Suisse de Zoologie, 41, 465-511.

Rithe, K. (2012). Spider diversity from relocated area of Melghat Tiger Reserve. Indian Journal of Arachnology, 1(2), 92-105.

Roy, T.K., Dhali, D.C., Saha, S., \& Raychaudhuri, D. (2010). Resurrection of the endemic bird dung crab spiders, Phrynarachne Thorell (Araneae, Thomisidae) of 19th century India. Munis Entomology \& Zoology, 5, 543-550.

Roy, T.K., Saha, S. and Raychaudhuri, D. (2017). Spider Fauna of Meghalaya, India. in, Biodiversity , Exploration, Exploitation, Conservation and Management - Vision and Mission. Proceedings of the UGC Sponsored National Seminar, Kolkata, India, 1920th November, 2016 (Eds. S. Saha, M. Manna, J. Ghosh, S. Podder, E. Haque, S. Guria and S. Dey). World Science News, 71, 104-127.

Saha, S., \& Raychaudhuri, D. (2004). Hitherto unknown genera of spiders, Ordgarius Keyserling, Pasilobus Simon (Araneidae) and Strigoplus Simon (Thomisidae) from eastern India. Journal of the Bombay Natural History Society, 101, 425-428.

Saha, S., \& Raychaudhuri, D. (2007a). Crab spiders (Araneae, Thomisidae) of Jaldapara Wildlife Sanctuary, Jalpaiguri, West Bengal - I. Journal of the Bombay Natural History Society, 104, 58-63. 
Saha, S., \& Raychaudhuri, D. (2007b). New crab spider of the genus Thomisus Walckenaer from Kolkata, West Bengal. Munis Entomology \& Zoology, 2, 439-442.

Saha, S., Bhadra, A., \& Raychaudhuri, D. (2020). Diversity spectrum of spider fauna in backyard rice agroecosystem, Narendrapur, West Bengal, India. World Science News, 147, 61-75.

Saha, S., Dhali, D.C., \& Raychaudhuri, D. (2015a). Spider fauna (Araneae, Arachnida) of Rajasthan with special reference to Ranthambore National Park, Rajasthan, India. Indian Journal of Arachnology, 4(1), 30-40.

Saha, S., Roy, T.K., Dhali, D.C. \& Raychaudhuri, D. (2015b). Spider faunal diversity of tea ecosystem of Assam, India. Romanian Journal of Bioloy \& Zoology, 60(1): 13-26.

Saha, S., Roy, T.K., \& Raychaudhuri, D. (2016). Survey on spider faunal diversity of Darjeeling tea plantations. Munis Entomology \& Zoology, 11(2), 622-635.

Sebastian, P.A., Murugesan, S., Mathew, M.J., Sudhikumar, A.V., Sunish, E. (2005a). Spiders in Mangalavanam, an ecosensitive mangrove forest in Cochin, Kerala, India (Araneae). European Arachnology-Acta Zoologica Bulgarica. No., 1, 315318.

Sebastian, P.A., Mathew, M.J., Beevi, S.P., Joseph, J., \& Biju, C.R. (2005b). The spider fauna of the irrigated rice ecosystem in central Kerala, India across different elevational ranges. Journal of Arachnology, 33(2), 247-255.

Sebastian, P.A., Mathew, M.J., \& Murugesan, S. (2011). Spider fauna in the forest and agricultural ecosystems of central Kerala, India. In, Arthropods and their Conservation in India (Insects \& Spiders) (Eds. Uniyal, V.P., \& Shrivastava, A.). ENVIS Bull. , Wildlife \& Protected Areas, 14, 159-174.

Sen, J.K. (1963). On a new species of the genus Thomisus Walckenaer, 1805, (Thomisidae, Arachnida) from India. Science and Culture, 29, 610612.

Sen, J.K. (1964). On a new spider of the genus Stiphropus Gerstaecker, 1873 from India (Thomisidae, Arachnida). Journal of the Zoological Society of India, 16, 65-67.

Sen, J.K., \& Basu, B.D. (1963). Thomisus mimae, a new species (Thomisidae, Arachnida) from Calcutta. Science and Culture, 29, 515-516.

Sen, S., Dhali, D.C., Saha, S., \& Raychaudhuri, D. (2015). Spiders (Araneae, Arachnida) of Reserve Forests of Dooars, Gorumara National Park, Chapramari Wildlife Sanctuary and Mahananda Wildlife Sanctuary. World Science News, 20, 1-339.

Sen, S., Saha, S., \& Raychaudhuri, D. (2009). Spiders of Ranthambore National Park, Rajasthan. Insect Environment, 16(4), 172-173.
Sen, S., Saha, S., \& Raychaudhuri, D. (2010a). A new spider genus of the tribe Smodicinini (Araneae, Thomisidae) from India. Munis Entomology \& Zoology, 5, 344-349.

Sen, S., Saha, S., \& Raychaudhuri, D. (2010b). Crab spiders (Araneae, Thomisidae) of Bandhavgarh National Park, with first record of Stiphropus sangayus Barrion \& Litsinger from India. Current Biotica, 4(3), 278-284.

Sen, S., Saha, S., \& Raychaudhuri, D. (2012). Addition to the crab spider (Araneae, Thomisidae) fauna of India. Munis Entomology \& Zoology, 7(2), 909-919.

Sharma, A., \& Singh, R. (2018a). Biodiversity and guild structure of spiders in northeastern Uttar Pradesh. Journal of Life Sciences, Bioinformatics, Pharmaceuticals \& Chemical Sciences, 4(4), 525-541.

Sharma, A., \& Singh, R. (2018b). Species diversity and guild structure of spiders from Siddharthnagar, Uttar Pradesh, India. Journal of Life Sciences, Bioinformatics, Pharmaceuticals \& Chemical Sciences, 4(4), 383-390.

Sharma, A., Singh, R., \& Singh, G. (2020a). Faunal diversity of Liocranidae, Mimetidae, Miturgidae, Nesticidae and Oecobiidae (Arachnida, Araneae) of India. Serket, 17(3), 270-283.

Sharma, A., Singh, G., \& Singh, R. (2020b). Faunal diversity of Linyphiidae (Araneomorphae, Araneae, Arachnida) in India. Asian Journal of Conservation Biology, 9(2), 304-314.

Sharma, A., Singh, G., \& Singh, R. (2021). Faunal diversity of spider families Dictynidae, Dysderidae, Eresidae and Filistatidae (Araneomorphae, Araneae, Arachnida) in India. International Journal of Zoology and Applied Biosciences, 6(1), 1-9.

Sharma, S., \& Ramakrishna, S. (2021). Study on diversity of spiders from Bangalore University Campus, Bengaluru, India. Int. J. Sci. Res., 10(3), 37-38.

Sherriffs, W.R. (1929). A contribution to the study of South Indian Arachnology IV. Annals and Magazine of Natural History, 10(2), 233-249.

Shraddha, K.K., Chaturved, S.R. (2019). A study on diversity of spiders at Malavagoppa village, in Shimoga District, Karnataka. International Journal of Environment Agriculture and Biotechnology, 4(2), 544-555.

Shraddha K.K., Chaturved, S.R. (2020). A Preliminary study on diversity of spiders at Amanikere Park in Tumakuru District, Karnataka. International Journal of Science and Research, 9(5), 570-581.

Siliwal, M., \& Molur, S. (2005). Range extension of Platythomisus sudeepi Biswas, 1977 (Araneae, Thomisidae). Zoos’ Print Journal, 20(5), 1871. 
Siliwal, M., Suresh, B., Dhuru, S., \& Pilo, B. (2003a). Spider diversity of riparian zone of river Vishwamitri, Gujarat. Journal of Current Science, 3(2), 429-434.

Siliwal, M., Suresh, B., \& Pilo, B. (2003b). Fauna of protected areas-3. Spiders of Purna Wildlife Sanctury, Dangs, Gujarat. Zoos’ Print Journal, 18(11), 12591263.

Siliwal, M., Molur, S., \& Biswas, B.K. (2005). Indian spiders (Arachnida, Araneae), updated checklist 2005. Zoos’ Print Journal, 20(10), 1999-2049.

Simon, E. (1885a). Matériaux pour servir à la faune arachnologiques de l'Asie méridionale.I.Arachnides recueillis à Wagra-Karoor près Gundacul, district de Bellary par M.M.Chaper.II.Arachnides recueillis à Ramnad, district de Madura par M.l'abbé Fabre. Bulletin de la Société Zoologique de France, 10, 1-39.

Simon, E. (1885b). Matériaux pour servir à la faune arachnologiques de l'Asie méridionale.III.Arachnides recueillis en 1884 dans la presqu'île de Malacca, par M.J.Morgan.IV.Arachnides recueillis à Collegal, district de Coimbatoore, par M.A.Theobald G.R. Bulletin de la Société Zoologique de France, 10, 436462.

Simon, E. (1895a). Histoire naturelle des araignées. Deuxième édition, tome premier. Roret, Paris, pp. 7611084.

Simon, E. (1895b). Descriptions d'arachnides nouveaux de la famille des Thomisidae. Annales de la Société Entomologique de Belgique, 39, 432-443.

Simon, E. (1906). Arachnides (2e partie). In, Voyage de M.Maurice Maindron dans l'Inde méridionale.8e Mémoire. Annales de la Société Entomologique de France, 75, 279-314.

Singh, B.B., \& Singh, R. (2014). Incidence and biodiversity of riceland spiders (Arthropoda, Arachnida) in northeastern Uttar Pradesh, India. Indo-American Journal of Life Sciences \& Biotechnology, 2(1), 64-89.

Singh, B.B., Singh, R., \& Singh, G. (2020). Faunal diversity of Clubionidae, Ctenidae, Cybaeidae, Deinopidae and Desidae (Araneomorphae, Araneae, Arachnida) in India. Journal of Applied Bioscience, 46(1, 2), 32-43.

Singh, B.B., Singh, R., \& Singh, G. (2021). Faunal diversity of spitting spiders (Scytodidae, Araneomorphae, Araneae, Arachnida) in India. World Journal of Pharmaceutical \& Life Sciences, 7(3), 8289.

Singh, R. (2021a). Distribution of Sparassidae (Araneomorphae, Araneae, Arachnida) in India. World Journal of Pharmaceuticals \& Life Sciences, 7(3), 134148.

Singh, R. (2021b). Faunal biodiversity of Tetragnathidae (Araneomorphae, Araneae, Arachnida) in India.
International Journal of Biological Innovations, 3(1), 92-119.

Singh, R. (2021c). Faunal biodiversity of Lycosidae (Araneomorphae, Araneae, Arachnida) in India, an updated checklist. International Journal of Zoological Investigations, 7(1), 110-158.

Singh, R. (2021d). Faunal diversity of Oxyopidae (Araneomorphae, Araneae, Arachnida) in India, an updated checklist. Journal of Global Bioscience, 10(4), 8539-8573.

Singh, R. (2021e). Faunal diversity of Theridiidae (Araneomorphae, Araneae, Arachnida) in India, An updated checklist. International Journal of Biological \& Environmental Investugations, 1(1), 1-35.

Singh, R., \& Singh, G. (2020). Diversity of mygalomorph spiders (Aranae, Opisthothelae) in India. International Journal of Biological Innovations, 2(2), 178-201.

Singh, R., \& Singh, G. (2021a). Faunal distribution of spiders of the families Titanoecidae, Trachelidae, Trochanteriidae, Uloboridae and Zodariidae (Arachnida, Araneae) in India. Serket, 17(4), 370-393.

Singh, R., \& Singh, G. (2021b). Updated checklist of Philodromidae (Araneae, Arachnida) from India. World Journal of Pharmaceuticals \& Life Sciences, 7(2), 129-139.

Singh, R., \& Singh, G. (2021c). Faunal diversity of Gnaphosidae (Araneomorphae, Araneae, Arachnida) in India: An updated checklist. Serket, 17(4), in press.

Singh, R., Singh, G., \& Sharma, A. (2020a). Diversity of yellow sac spiders (Cheiracanthiidae, Araneae, Arachnida) in India. Journal of Entomology and Zoology Studies, 8(6), 118-126.

Singh, R., Singh, G., \& Sharma, A. (2020b). Faunal diversity of Hahniidae, Hersiliidae and Homalonychidae (Arachnida, Araneae, Araneomorphae) in India. Sertket, 17(3), 240-251.

Singh, R., Singh, G., \& Singh, B.B. (2020c). Diversity of Asemoneinae, Eupoinae, Hisponinae, Lyssomaninae, Onomastinae and Spartaeinae (Arachnida, Araneae, Salticidae) in India, a checklist and bibliography. Research Journal of Life Sciences, Bioinformatics, Pharmaceuticals \& Chemical Science, 6(5), 29-46.

Singh, R., Singh, G., \& Singh, B.B. (2020d). Diversity of Amycoida and Astioida (Arachnida, Araneae, Salticidae, Salticinae) in India. Journal of Entomology and Zoology Studies, 8(5), 1478-1488.

Singh, R., Singh, G., \& Singh, B.B. (2020e). Diversity of Marpissoida, Chrysillini and Hasariini (Arachnida, Araneae, Salticidae, Salticinae) in India. Research Journal of Life Sciences, Bioinformatics, Pharmaceuticals \& Chemical Science, 6(6), 15-42.

Singh, R., Singh, G., \& Singh, B.B. (2020f). Diversity of simonid spiders (Araneae, Salticidae, Salticinae) in 
India. International Journal of Biological Innovations, 2(2), 247-276.

Singh, R., Singh, G., \& Singh, B.B. (2021a). Faunal diversity of jumping spiders (Salticidae, Araneae, Arachnida) in India. International Journal of Biological Innovations, 3(1):1-37.

Singh, R., Singh, G., \& Singh, B.B. (2021b). Faunal diversity of Agelenidae, Amaurobiidae, Anyphaenidae, Arkyidae, Cithaeronidae and Corinnidae (Araneae, Arachnida) in India. Munis Entomology \& Zoology, 16(2), 772-786.

Singh, S., Borkotoki, A., \& Sarmah, C.K. (2012). Species distribution of spiders in barpeta district of Assam, a diversity measure. International Science Research Journal, 4(1), 47-57.

Sivaperuman, C., \& Rathore, N.S. (2004). A preliminary report on spiders in Desert National Park, Rajasthan, India. Zoos’ Print Journal, 19(5), 1485-1486.

Smitha, M.S., \& Sudhikumar, A.V. (2020). A diversity of spiders (Arachnida, Araneae) from a cashew ecosystem in Kerala, India. Journal of Threatened Taxa, 12(13), 16879-16884.

Solanki, R., \& Kumar, D. (2014). Effect of pesticides on spider population in cotton Agro-system of Vadodara (Gujarat). The IIS University Journal of Science \& Technology, 3(1), 48-52.

Solanki, R., Siliwal, M., \& Kumar, D. (2020). A preliminary checklist of spiders (Araneae, Arachnida) in Jambughoda Wildlife Sanctuary, Panchmahal District, Gujarat, India. Journal of Threatened Taxa, 12(11), 16576-16596.

Stoliczka, F. (1869). Contribution towards the knowledge of Indian Arachnoidea. Journal of the Asiatic Society of Bengal, 38(2), 201-251.

Sudhikumar, A.V., Mathew, M.J., Sunish, E., Murugesan, S., \& Sebastian, P.A. (2005). Preliminary studies on the spider fauna in Mannavan shoal forest, Kerala, India (Araneae). European Arachnology-Acta Zoologica Bulgarica, Suppl. No., 1, 319-327.

Sugumaran, M.P., Soundararajan, R.P., \& Lakshamanan, V. (2007). Spider fauna in the horticultural crops of Yercaud hills. Zoos’ Print Journal, 22(6), 2721-2722.

Sumesh, N.V., \& Sudhikumar, A.V. (2020). Checklist of spiders from the sacred groves of Northern Kerala, India. Uttar Pradesh Journal of Zoology, 41(9), 104115.

Sunil Jose, K., \& Sebastian, P.A. (2001). New report on some crab spiders (Araneae, Thomisidae) from Kerala, India. Entomon, 26, 183-189.

Sunil Jose, K., Samson, D., Sudhikumar, A.V., Sebastian, P.A. (2003). Description of Female Amyciaea forticeps (Cambridge), Araneae, Thomisidae, with a
Redescription of Its Male from Kerala, India. Journal of the Bombay Natural History Society, 100, 157-160.

Sunil Jose, K., Sudhikumar, A.V., Davis, S., \& Sebastian, P.A. (2008). Preliminary studies on the spider fauna (Arachnida, Araneae) in Parambikulam Wildlife Sanctuary in Western Ghats, Kerala, India. Journal of the Bombay Natural History Society, 105(3), 264-273.

Tabasum, N.R., Nagaraj, B, Shantakumari, S., Sreenivasa, V., \& Sai Sandeep, Y. (2018). Assessment of spider diversity and composition along the Tungabhadra Irrigation Channel at Ballari, Karnataka. International Journal of Biological Science, 9(1), 36-44.

Tang, G., Yin, C., \& Peng, X. (2009). Six crab spiders of the subfamily Stephanopinae from southeast Asia (Araneae, Thomisidae). The Raffles Bulletin of Zoology, 57(1), 39-50.

Thorell, T. (1891). Spindlar från Nikobarerna och andra delar af södra Asien. Kongliga Svenska VetenskapsAkademiens Handlingar, 24(2), 1-149.

Tikader, B.K. (1960). On some new species of spiders (Arachnida) of the family Thomisidae from India. Journal of the Bombay Natural History Society, 57, 173-183.

Tikader, B.K. (1961). On two new species of spider of the genus Oxyptila (family, Thomisidae) from India. Proceedings of the Zoological Society, Calcutta , 13, 115-118.

Tikader, B.K. (1962). Studies on some Indian spiders (Araneae, Arachnida). Journal of the Linnean Society of London, Zoology, 44(300), 561-584.

Tikader, B.K. (1963). Studies on interesting south Indian crab-spiders (Family, Thomisidae). Proceedings of the Indian Academy of Science, 58(B), 249-262.

Tikader, B.K. (1964). A new species of spider of the genus Oxyptila (family Thomisidae) from India. Science and Culture, 30, 152-153.

Tikader, B.K. (1965). On some new species of spiders of the family Thomisidae from India. Proceedings of the Indian Academy of Science, 61(B), 277-289.

Tikader, B.K. (1966a). Studies on some crab-spider (family, Thomisidae) from Khasi and Jaintia hills, Assam, India. Proceedings of the Indian Academy of Science, 64(B), 53-61.

Tikader, B.K. (1966b). Description of two new species of the genus Xysticus (family, Thomisidae) from India. Journal of the Asiatic Society of Bengal, 8, 249-252.

Tikader, B.K. (1968). Studies on spider fauna of Khasi and Jaintia hills, Assam, India. Part-II. Journal of the Assam Science Society, 10, 102-122.

Tikader, B.K. (1970). Spider fauna of Sikkim. Records of the Zoological Survey of India, 64, 1-83. 
Tikader, B.K. (1971). Revision of Indian crab spiders (Araneae, Thomisidae). Memoirs of the Zoological Survey of India, 15(8), 1-90.

Tikader, B.K. (1977). Studies on spider fauna of Andaman and Nicobar islands, Indian Ocean. Records of the Zoological Survey of India, 72, 153-212.

Tikader, B.K. (1980). Fauna of India, Aranae, Vol.1, Part 1, Thomisidae (Crab-spiders). Zoological Survey of India, Kolkata, pp. 1-247.

Tikader, B.K., \& Biswas, B. (1974). Some spiders of the genus Xysticus (family, Thomisidae) from Darjeeling, India. Proceedings of the Indian Academy of Science, 80(B), 262-266.

Tikader, B.K., \& Biswas, B. (1979). Two new species of spider of the genus Tharpyna Koch from India (Family, Thomisidae). Journal of the Bombay Natural History Society, 75, 903-905.

Tikader, B.K., \& Biswas, B. (1981). Spider fauna of Calcutta and vicinity, Part-I. Records of the Zoological Survey of India, Occasional Paper No., 30, 1-149.

Tiwari, A.K., \& Singh, R. (2021). Diversity and distribution of Pisauridae (Araneae, Araneomorphae, Arachnida) in India. International Journal of Entomology Research, 6(1), 119-125.

Tiwari, A.K., Singh, G., \& Singh, R. (2021a). Faunal diversity of Oonopidae (Araneomorphae, Araneae, Arachnida) in India. Journal of Global Biosciences, 10(1), 8340-8351.

Tiwari, A.K., Singh, G., \& Singh, R. (2021b). Biodiversity of some poorly known families of spiders (Areneomorphae, Araneae, Arachnida) in India. Journal of Global Biosciences, 10(1), 8352-8371.

Tiwari, A.K., Singh, R., \& Singh, G. (2021c). Diversity and distribution of Pholcidae (Araneae, Araneomorphae, Arachnida) in India. International Journal of Life Sciences, 9(1), 124-134.

Trivedi, V. (2009). Diversity of spiders in groundnut crop fields in village area of Saurashtra region. Journal of the Bombay Natural History Society, 106(2), 184-189.

Uniyal, V.P. (2006). Records of spiders from Indian transHimalayan region. Indian Forester, 132(12a), 177-181.

Uniyal, V.P., \& Hore, U. (2006). Studies on the spider fauna in mixed sal forest area of Chandrabani, Dehradun. Indian Forester, 132(12a), 83-88.

Uniyal, V.P., \& Hore, U. (2009). Effect of management practices on spider diversity in Terai Conservation Area (TCA). Final Project Report, Wild Life Institute of India. pp. 222.

Uniyal, V.P., Sivakumar, K., \& Quasin, S. (2011). Diversity of spiders in Nanda Devi Biosphere Reserve.
Wildlife Institute of India, Dehradun. DST Project Completion Report, pp. 199.

Vaibhav P.U, Vidyavati M.H, Tanuja K.D., Milind F.N., Karuna G., Veeranagoudar D.K., \& Pulikeshi M.B. (2017). Spider diversity of Karnatak University Campus, Dharwad. International Journal of Advanced Scientific Research and Management, 2(1), 12-26.

Vairale A.B, Wagh G.A. (2021). Diversity of spiders in microhabitats of a tropical reserve forest of Amravati, Maharashtra, India. Bioscience Biotechnology Research Communications, 14(1), 446-452.

Vineetha, VP \& George, J. (2021). Diversity of spiders (Araneae, Arachnida) in selected mangrove ecosystems of Northmalabar, Kerala, India. International Journal of Entomology Research, 6(2), 170-174.

Warghat, N., Sharma, N., Thakur, P., \& Baig, M. (2014). Genetic diversity analysis of crab spider (Araneae, Thomisidae) based on RAPD-PCR. Indian Journal Applied Research, 4(8), 1-5.

Warghat, N.E., Gaur, A.J., Sharma, N.R., Chirde, S.G., \& Chandrakar, M.R. (2011). Spiders (Araneae) from agricultural fields near foothill of Satpura Mountain ranges of Amravati District, Maharashtra, India. Nature Precedings. 1-11. doi:10.1038/npre.2011.5912.1

White, L., Pryce, D., Wilkins, V.L., \& Dutton, A.J. (2019). Bonapruncinia sanctaehelenae. The IUCN Red List of Threatened Species 2019: e.T65881343A67528211.

WSC (2021). World Spider Catalog. Version 22.0. Natural History Museum Bern, online at http://wsc.nmbe.ch, accessed on 10 July, 2021.

Yadav, A. (2019). Diversity and ecology of spiders in Champaner-Pavagadh Archaeological Park, a world heritage site in Gujarat. Ph. D. Thesis, The Maharaja Sayajirao University of Baroda, Vadodara, Maharashtra, India.

Yadav, A., Solanki, R., Siliwal, M., \& Kumar, D. (2017a). Spiders of Gujarat, a preliminary checklist. Journal of Threatened Taxa, 9(9):10697-10716.

Yadav, M., Goswami, T.N., Anil \& Ray, S.N. (2016). Species composition of spider-fauna in paddy ecosystem throughout the cropping period at Sabour, Bihar, India. Ecology, Environment and Conservation, 22(2), 719-722.

Yadav, S., Patil, V., \& Ismavel, V.A. (2017b). Report of Platythomisus octomaculatus (C.L.Koch, 1845) and Platythomisus sudeepi Biswas, 1977 from India (Araneae, Thomisidae). Biodiversity Data Journal, 5(e10294), 1-8. 